\title{
Efficacy of Collagenase Clostridium Histolyticum for Dupuytren Disease: A Systematic Review
}

\section{Eficacia de la colagenasa clostridium histolyticum para la enfermedad de dupuytren: revision sistematica}

\author{
Francisco Javier Carrera-Hueso ${ }^{4}$

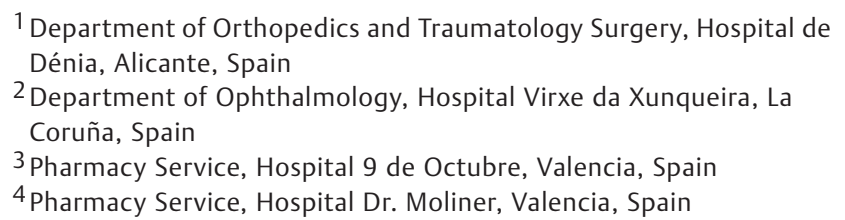

Rafael Sanjuan-Cerveró ${ }^{1}$ Pedro Vazquez-Ferreiro ${ }^{2}$ Diego Gomez-Herrero ${ }^{3}$

Address for correspondence Rafael Sanjuan Cerveró, MS, Department of Orthopedics and Traumatology Surgery, Hospital de Dénia, Partida de Beniadla s/n, 03700, Dénia, Alicante, Spain (e-mail: sanjuan.rafcer@gmail.com).

Rev Iberam Cir Mano 2017;00:70-88.

received

July 26, 2017

accepted

September 19, 2017

published online

November 22, 2017
Introduction Collagenase Clostridium Histolyticum $(\mathrm{CCH})$ has become a therapeutic alternative for Dupuytren disease. However, its efficacy in the medium to long term is unknown. The objective of our study is to carry out a systematic review of the studies conducted on the subject.

Material and Methods Systematic bibliographic search. Analysis depending on the time of progression, looking into 2 groups with the follow-up cut-off point of 1 year. Analysis of the number of patients who reached the primary endpoint, of the mean correction in degrees, and of the proportional correction of each joint.

Results The 50 selected clinical trials encompass a total of 4,622 patients (an average of 92.70). A total of 7,546 joints were treated with the mean being 148.15 joints per trial (3,925 metacarpophalangeal [MCP] and 2,350 proximal interphalangeal [PIP]). In less than one year of progression, the primary end point was reached in $48.9 \%$ of the joints ( $69.77 \%$ of the MCPs and $30.14 \%$ of the PIPs), the mean correction in degrees was 45.5 (standard deviation [SD]: 19.18) degrees; 40.8 degrees in the MCP (SD: 10.12) and 35.6 in the PIP (SD: 13.23), and the proportional correction of the joints was $72.9 \%$ (SD: 14.43) (83.9\% for MCPs [SD: 12.58] and 64.2 for the PIPs [SD: 16.35]). In the follow-ups over 1 year, the primary end point was reached at a rate of 57.5\% (68.9\% of the MCPs and $43.3 \%$ of the PIPs), the mean correction in degrees was 37.6 degrees (SD: 10.93) (37.3 degrees in the MCPs [SD: 9.98] and 23.7 in the PIPs [SD: 16.33]) and the proportional correction of the joints was $87.3 \%$ (SD: 10.96) (90.3\% for MCP [SD: 6.94] and 75\% for PIP [SD: 13.54]).

- systematic review

DOI https://doi.org/

10.1055/s-0037-1608749. ISSN 1698-8396.
Copyright $\odot 2017$ Thieme Revinter

Publicações Ltda, Rio de Janeiro, Brazil
License terms

(c) $(1) \$$ 


\section{Resumen}

\author{
Palabras Clave \\ - colagenasa \\ - clostridium \\ histolyticum \\ - eficacia \\ - revision \\ sistematica
}

Conclusions The results indicate a satisfactory response to $\mathrm{CCH}$ treatment maintained in the short and medium term. The recurrence rate is uncertain given the available data.

Introducción La Colagenasa Clostidium Histolyticum se ha convertido en una alternativa terapéutica para la enfermedad de Dupuytren. Sin embargo, se desconoce su eficacia a medio y largo plazo. El objetivo de nuestro trabajo consiste en la realización de una revisión sistemática de los trabajos realizados.

Material y Método Búsqueda bibliográfica sistemática. Análisis dependiendo del tiempo de evolución considerándose dos grupos con el punto de corte de seguimiento un año. Análisis del número de pacientes que alcanzaron el objetivo primario, la corrección media en grados y la alcanzada por cada articulación.

Resultados Los 50 estudios clínicos elegidos, engloban un total de 4622 pacientes (media de 92.70). Se ha tratado un total de 7546 articulaciones siendo la media 148.15 (3925 MCF y 2350 IFP). A menos de un año de evolución el Primary End Point se alcanzó en el $48,9 \%$ de las articulaciones (69,77\% de las MCF y $30,14 \%$ de las IFP), la corrección media en grados fue de 45.5 (DE: 19.18) grados; 40.8 grados en las MCF (DE: 10.12) y 35.6 en las IFP (DE: 13.23) y la corrección proporcional de las articulaciones fue del $72,9 \%$ (DE: 14.43$)(83,9 \%$ para las MCF (DE: 12.58$)$ y 64.2 para las IFP (DE: 16.35$)$. En seguimientos mayores a un año el Primary End Point se alcanzó en el 57,5\% (68,9\% de las MCF y $43,3 \%$ de las IFP), la corrección media en grados fue de 37.6 grados (DE: 10.93 ) (37.3 grados en las MCF (DE: 9.98) y 23.7 en las IFP (DE: 16.33) y la corrección proporcional de las articulaciones fue del $87,3 \%$ (DE: 10.96) (90,3\% para las MCF (DE: 6.94) y un $75 \%$ de las IFP (DE: 13.54).

Conclusiones Los resultados indican una respuesta satisfactoria al tratamiento con $\mathrm{CCH}$ mantenida a corto y medio plazo. La tasa de recurrencias es un dato incierto con los datos disponibles.

\section{Introduction}

The collagenase clostridium histolyticum $(\mathrm{CCH})$ used for Dupuytren Disease (DD) is a mixture of two enzymes that degrade collagen types I and III found in the abnormal tissue that constitutes this fibromatosis. The first attempt to inject substances inside the cord related to DD was made by Bassot, ${ }^{1,2}$ in the 1960s. He coined the term "pharmacodynamical exeresis" and obtained relatively good results, published in $1969^{3}$, looking for a proteolytic, anti-inflammatory and anesthetic effect. The term "enzymatic aponeurotomy" was adopted in 1971 by Hueston ${ }^{3}$, who slightly modified the mixture for the injection. The treatment is identified as a valid alternative for patients who cannot be treated by the usual means. Using a similar technique, McCarthy obtained good results suggesting that the technique is an effective alternative as a substitute for fasciectomy. ${ }^{2}$ Finally, the studies by Hurst and Badalamente ${ }^{4,5}$ established the efficacy of the treatment with an enzyme, the $\mathrm{CCH}$, that allowed to break the cord in a local and minimally invasive manner.

Since the publication of the first clinical trial with $\mathrm{CCH}^{6}$ and its marketing in the USA and Europe, this drug has become more important over time ${ }^{7,8}$ with regard to the treatment of DD. The development of the CORDLESS clinical trial, ${ }^{9,10}$ which involved the follow-up of patients enrolled in four previous clinical trials, has improved the level of understanding of progression in the short and medium terms. Likewise, these studies have been the basis of other publications regarding the analysis of subgroups ${ }^{11}$ and partial results. ${ }^{12}$

Numerous clinical series and comparative studies have been published since then, providing an independent point of view regarding the clinical results obtained with the treatment. The variability among them is the norm rather than the exception, and the comparability between studies is a complex matter. ${ }^{13}$ Although $\mathrm{CCH}$ is nowadays an alternative adopted by numerous hand surgeons in the treatment of DD, the rate of recurrence is unknown; thus, its status in the therapeutic arsenal compared with surgery is not clear. The scientific evidence at this time is also limited since no systematic reviews have been performed so far, and comparative studies with other techniques have been limited. The objective of our study is to carry out a systematic review covering all these studies, which evaluate independent studies and demonstrates the result of the $\mathrm{CCH}$ treatment since its commercialization aimed at assessing the efficacy of the treatment.

\section{Material and Methods}

A structured bibliographic search was performed in the PubMed, Google Scholar, Ovid and Web of Science databases 
with the following strategy: (Dupuytren disease [MeSH Terms]) AND (collagenase [MeSH Terms] AND clostridium histolyticum [MeSH Terms]). The search covered articles published from September 3, 2009 (date of publication of the CORD I study ${ }^{6}$ ) until June 15, 2017. A search of gray literature was performed in the databases of doctoral theses and at the US National institutes of Health Clinical Trials (www.clinicaltrials.gov), as well as through a manual review of the bibliography included in the articles.

We initially included all designs of cohort studies, clinical trials, case-control and case series published in English, Spanish, German, French and Italian; and those in which patient follow-up was specified for at least 30 days. Only studies that included patients diagnosed with Dupuytren contracture susceptible to surgical intervention with an initial contracture degree equal to or greater than $20^{\circ}$, and with at least one group of patients treated with $\mathrm{CCH}$ were considered. Reanalyzes of previous series, or those that did not provide data for the analysis, were excluded.

A structured form was used for the extraction and definitive collection of the data from the studies selected independently by two authors. The discrepancies that emerged upon comparing the results of both authors were resolved by a third author. To assess bias in the studies, we used the strategic orientation of business enterprises (STROBE) scale, with two researchers scoring each of the selected studies and using the mean of the two to assess their quality. Any disagreements were resolved through consensus with a third researcher.

The main variables collected from each study were the features and the design of the study, the interventions performed, the $\mathrm{CCH}$ doses utilized, the number of injections per patient, the extension time, and the criteria regarding severity and results, as well as the follow-up time. Finally, the funding from each study, if any, was collected. In clinical trials and comparative observational studies, only the clinical results of the group of patients treated with $\mathrm{CCH}$ were taken, and they were taken as a sample reference.

An analysis of the existing literature was conducted with regard to the results, taking into consideration two groups with a follow-up cut-off of 1 year. The clinical results were assessed based on three parameters: A) The number of patients who have reached the primary end point established in the CORD studies ${ }^{6,14}$ (final extension after treatment between $0-5^{\circ}$ ) stating the result in total absolute percentage of joints that have reached the objective; $\mathrm{B}$ ) The assessment of the mean correction of each joint in degrees was evaluated, defined as the result of the degree of initial contracture minus the degree of final contracture; and C) Data regarding the correction ratio of the treated joint was also collected. If an article stated the results of two different modes, both have been included. An assessment of the recurrences over time has been made in the studies intended for that purpose, ${ }^{9,10}$ as well as in all those that cited them in their series. Finally, the concept of "noneffective" treatment in the series has been assessed. The missing data in the tables explain the lack of direct correlation between results $(A+B \neq C$, as in one study overall results may have been given and not the results related to the metacarpophalangeal $[\mathrm{MCP}]$ and proximal interphalangeal [PIP] joints, for example).

The calculation of the results stated in the text has been performed as follows. The primary end point included in the CORD studies ${ }^{6,14}$ was established as the primary reference for the results. The secondary end point is similarly indicated in the results of the CORD studies, ${ }^{6,14}$ such as the patients who showed at least $50 \%$ of improvement since the initial contracture. In our analysis, the reflected number of these patients is that of those who have reached this point, while those included in the primary end point have been excluded, thus specifying the patients with improvement greater than $50 \%$, but excluding those who have reached extensions between $0-5$ degrees. The mean correction of the affected joints was obtained by acquiring direct data from the corresponding article, or the difference between the initial mean degrees of the middle and the endpoints. Finally, the proportion or percentage of the correction achieved was obtained in the same way, indicating the correction percentage for each of the treated joints.

The immediate measures to be implemented following treatment with $\mathrm{CCH}$ is a controversial issue. Therefore, in the articles analyzed, usage of orthosis and referrals to a physiotherapy protocol following $\mathrm{CCH}$ administration have been reviewed. In the final chapters, we analyzed both the recurrences and the treatment failures, commonly considered in the literature as "non-responders."

\section{Results}

\section{Search Results}

Total 598 articles were obtained, of which only 240 studies have met the inclusion criteria. After removing duplicates, the elimination followed the exclusion criteria following the reading of the review summary, clinical cases, letters to the director, editorials and meeting summaries. Total 61 articles were obtained for analysis. These studies were reviewed through a complete reading of the article. Eleven studies were eliminated for a variety of reasons: 6 due to insufficient data on clinical outcomes (including cost studies), 3 due to cross-references to previous studies (subgroup analysis), and 2 due to exclusive references to results related to thumbs (excluded from the analysis of the CORD study ${ }^{6,14}$ ). In the end, 50 publications were analyzed (-Fig. 1). The interobserver assessment performed using the STROBE scale of the included articles has shown great homogeneity among the researchers (kappa $>0.85$ ).

\section{Results of the Clinical Studies}

The main features of the selected studies are shown in -Table 1. The monitoring of progression corresponding to the 3-year CORDLESS study has been included in the series, ${ }^{9}$ taking into account that the patients associated with the CORD I, CORD II, POINT I and POINT II studies have been excluded for the calculation of demographic data. ${ }^{6,14,15}$ The POINT X study was also excluded ${ }^{16}$ because it was conducted in a subgroup of patients associated with 


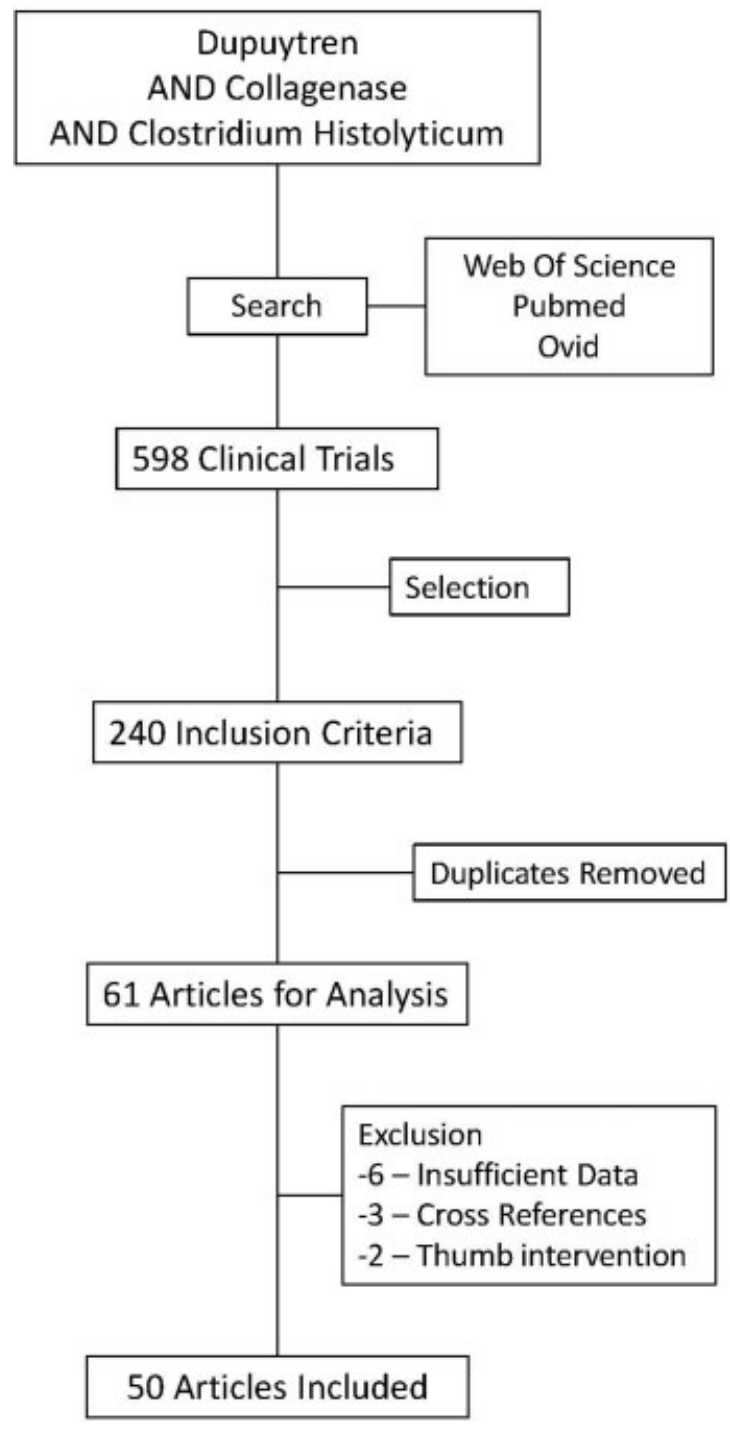

Fig. 1 Flowchart of the selection of articles analyzed.

the European POINT II study. The median follow-up of patients was 265 days (range 14-1,095; standard deviation [SD]: 233 days). The mean number of patients per study was 95 with a very variable range between $714^{17}$ and 8 , in the first independent series published in Germany ${ }^{18}$ (- Table 2).

The mean age of the patients was 65.2 years (range 61.070.0). Male patients corresponded to $85 \%$ of the patients in the studies (mean 92.5, range 7-542). Thirty-three series were developed in a single center and 18 studies were multicenter projects. With regard to the temporal evolution, 36 studies were prospective and 15 were retrospective.

We have found a great heterogeneity of studies with many methodological variations. The two clinical trials corresponded to the CORD I ${ }^{6}$ and CORD II $^{14}$ clinical trials performed in the USA and Australia, respectively, and which compared the effect of $\mathrm{CCH}$ over placebo. The nine casecontrol series compared the effect of $\mathrm{CCH}$ with that of surgery through fasciectomy ${ }^{19-23}$ or with needle aponeurotomy. ${ }^{24-27}$ Even though most of the studies were based on clinical series with a time-based follow-up, they ranged from cost studies, ${ }^{28,29}$ implementation of treatment in selective population series, ${ }^{30,31}$ physiotherapy protocols as a comparative group, ${ }^{32}$ assistance with ultrasound treatment ${ }^{33}$ or the selective reimplementation of treatment to patients who had undergone a previous treatment with $\mathrm{CCH}^{34}$ Two studies were limited to assessing the treatment of a single joint of the same finger comparing it with aponeurotomy: Stromberg, ${ }^{25}$ on the MCP, and Skov, ${ }^{27}$ on the PIP. Skirven ${ }^{32}$ performed his study only with PIP results.

As for the study methodology, we have found different models of study design, such as case study series with different programs; ${ }^{35,36}$ open label studies, such as JOINT ${ }^{15}$ or ReDUCTo, ${ }^{37}$ or studies such as CORD II, ${ }^{14}$ that begin as double blind randomized and continue as open label, from which only the data from the first part was collected to include the results of the second part in the CORDLESS study. ${ }^{9}$ Warwick ${ }^{38}$ performed a series in which results regarding "unusual" cords (natatory, Y-shaped or crow-foot cords) were specified in the same way as Verheyden. ${ }^{39}$ Several studies ${ }^{40-42}$ assessed the impact with respect to the extension time following consistent administration of treatment.

The inclusion and exclusion criteria for the studies included were based on the drug's fact sheet. All patients included presented with a minimum initial contracture of $20^{\circ}$ in the PIP or MCP joints. Studies that have strictly followed the CORD criteria have limited the maximum degree of flexion also included in the study. The follow-up criteria showed a great deal of heterogeneity. After reading the articles, 26 studies, directly or indirectly, followed the criteria of the CORD clinical trials to demonstrate their results. On the other hand, 5 followed the Tubiana classification, ${ }^{18,28,43-45}$ and 1 followed its own criteria. ${ }^{30}$ The remaining 19 have not specified their follow-up criteria. The measurement of results has mixed values at varying degrees of measuring: a priori 16 articles used the range of movement (ROM) to evaluate results in whole or in part, while the remaining 35 did not use this method, limiting themselves to assessing extension deficit results in degrees by separate joints, groups of joints (passive extension deficit [PED]), or one article even ${ }^{44}$ includes the PIP (total passive extension deficit [TPED]).

The 50 selected clinical trials encompass a total of 4,622 patients (mean 92.70). A total of 7,546 joints were treated with an average of 148.15 joints treated per study $(3,925$ MCP [ 10.31 on average] and 2,350 PIP [58.03 on average]). The administered dose was standard in all studies, except for two studies that used double dose on the same hand at a time, ${ }^{17,46}$ and four that used the standard dose plus the amount remaining in the vial in various formats. ${ }^{39,47-49}$ Twenty-three studies followed an injection protocol in which they allowed one to three infiltrations per joint and patient, four ${ }^{25,29,38,47}$ studies used one or two injections, even though they followed the CORD protocols, and presumably if it were needed, they would have used three, and the rest used a protocol of one injection for an infiltration of a joint. In the studies that specified how many vials were used per joint, the mean was 1.23 (range 0.8-1.6; SD: 0.36), with only one study having used less than one vial per joint, ${ }^{50}$ and with 


\begin{tabular}{|c|c|c|c|c|c|c|c|c|c|c|c|c|c|c|c|c|c|c|c|c|c|}
\hline 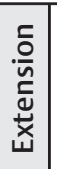 & $\stackrel{\sim}{\sim}$ & $\stackrel{\searrow}{\sim}$ & $\begin{array}{c}\stackrel{r}{m} \\
1 \\
\dot{\sim}\end{array}$ & $\stackrel{\beth}{\sim}$ & & $\stackrel{\searrow}{\sim}$ & & $\stackrel{\searrow}{\sim}$ & $\stackrel{\searrow}{\sim}$ & $\stackrel{\sim}{\sim}$ & $\stackrel{\Delta}{\sim}$ & $\stackrel{\Delta}{\sim}$ & $\stackrel{\searrow}{\sim}$ & $\stackrel{\nabla}{\sim}$ & $\stackrel{\searrow}{\sim}$ & $\stackrel{ \pm}{\sim}$ & 1 & $\stackrel{\ddagger}{\sim}$ & & $\begin{array}{l}\infty \\
\stackrel{\infty}{1} \\
\stackrel{+}{\sim}\end{array}$ & $\stackrel{\searrow}{\sim}$ \\
\hline 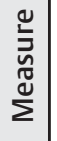 & $\underset{\substack{0 \\
\propto}}{2}$ & $\sum_{\substack{0 \\
\hdashline}}$ & $\begin{array}{l}\frac{\tilde{y}}{0} \\
\vec{x} \\
\vec{x}\end{array}$ & $\sum_{\propto}$ & $\sum_{\propto}$ & 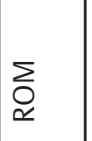 & $\sum_{\substack{0 \\
\propto}}$ & 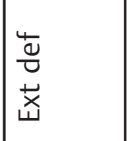 & 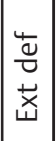 & 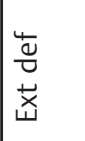 & $\mid$\begin{tabular}{|l|} 
\\
\\
$\vec{v}$ \\
$\vec{x}$ \\
$\vec{v}$
\end{tabular} & 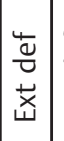 & 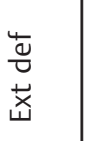 & 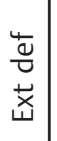 & $\sum_{0}$ & $\sum_{2}$ & 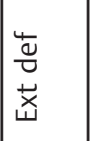 & \begin{tabular}{|l|} 
\\
$\underline{y}$ \\
$\vec{x}$ \\
$\vec{w}$
\end{tabular} & $\begin{array}{l}\frac{4}{\tilde{c}} \\
\vec{x} \\
\vec{x}\end{array}$ & $\underset{\substack{0 \\
\propto}}{2}$ & 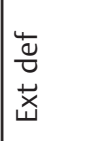 \\
\hline 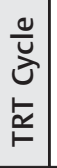 & {$\left[\begin{array}{l}\stackrel{\mathcal{J}}{f} \\
1\end{array}\right.$} & $\underline{m} \stackrel{1}{1}$ & 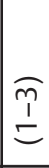 & - & $\underline{m}$ & $\begin{array}{ll}\infty & \bar{m} \\
0 & \underline{1} \\
r & =\end{array}$ & & - & - & & - & - & $\stackrel{m}{!}$ & - & 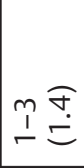 & - & - & - & & 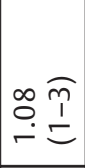 & $\stackrel{\widehat{m}}{\stackrel{1}{=}}$ \\
\hline 亗 & $\backsim$ & $\backsim$ & $\backsim$ & $\backsim$ & $\backsim$ & $\backsim$ & $\backsim$ & $\backsim$ & $\backsim$ & $\backsim$ & $\backsim$ & $\backsim$ & $\backsim$ & $\backsim$ & $\backsim$ & $\backsim$ & $\backsim$ & $\backsim$ & $\backsim$ & $\backsim$ & $\backsim$ \\
\hline 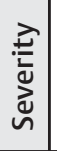 & 命 & 号 & 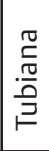 & 产 & 命 & 命 & & 策 & 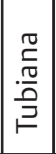 & & 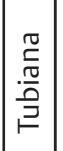 & 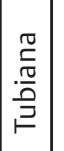 & & 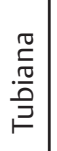 & 号 & $\begin{array}{l}\text { Oे } \\
\text { 宅 }\end{array}$ & & & & 产 & 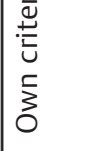 \\
\hline 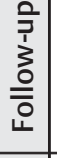 & ঃ & ৪ & $\stackrel{\square}{ }$ & $\stackrel{尺}{\curvearrowright}$ & 岁 & $\stackrel{\circ}{m}$ & 脶 & $\stackrel{m}{m}$ & $\stackrel{m}{\circ}$ & 号 & $\left(\begin{array}{l}\infty \\
\hdashline\end{array}\right.$ & $\left|\begin{array}{l}n \\
0 \\
m \\
m\end{array}\right|$ & 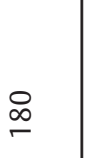 & $\stackrel{\infty}{\triangleright}$ & $\stackrel{?}{\stackrel{\circ}{N}}$ & $\begin{array}{l}n \\
\end{array}$ & $\stackrel{一}{m}$ & 聍 & $\begin{array}{l}n \\
\stackrel{n}{n}\end{array}$ & $\underset{\mathscr{F}}{\stackrel{\sim}{\sim}}$ & $\begin{array}{l}\hat{\sigma} \\
\text { r }\end{array}$ \\
\hline 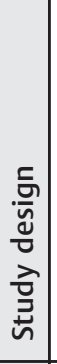 & 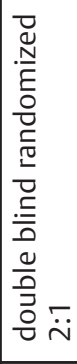 & 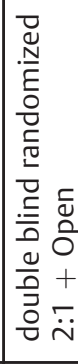 & 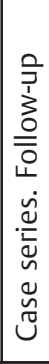 & 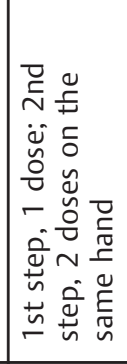 & 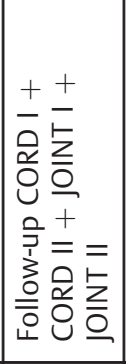 & 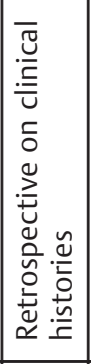 & 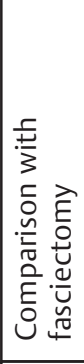 & 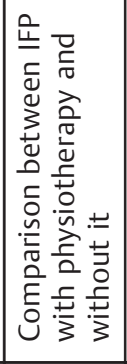 & $\begin{array}{l}0 \\
\overline{1} \\
3 \\
0 \\
\overline{0} \\
4 \\
\end{array}$ & 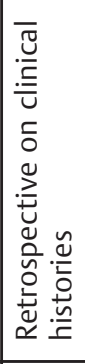 & 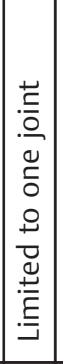 & 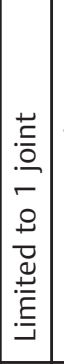 & 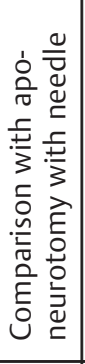 & 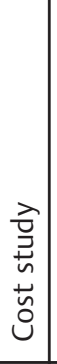 & ळ & 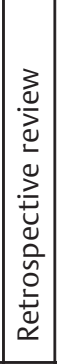 & 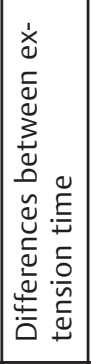 & 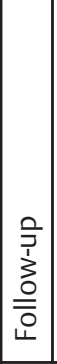 & $\begin{array}{l}0 \\
7 \\
\vdots \\
0 \\
0 \\
\end{array}$ & 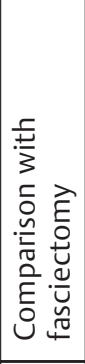 & 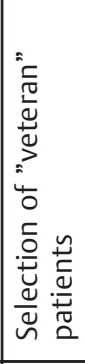 \\
\hline 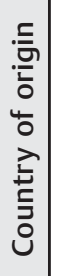 & 芴 & 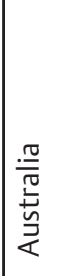 & 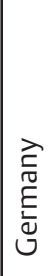 & 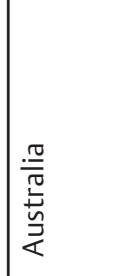 & 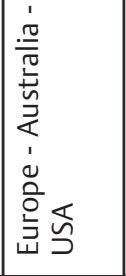 & 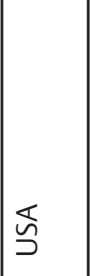 & 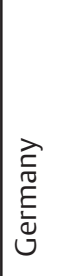 & 芯 & $\begin{array}{l}\frac{5}{\pi \tilde{0}} \\
\text { กิ }\end{array}$ & 芯 & $\mid \begin{array}{l}\overrightarrow{\hat{T}} \\
\pm\end{array}$ & 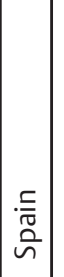 & $\overleftarrow{\nwarrow}$ & $\begin{array}{l}. \frac{5}{\pi} \\
\tilde{n}\end{array}$ & 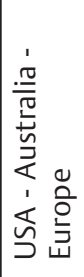 & 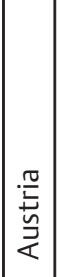 & 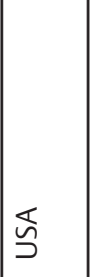 & 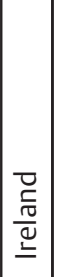 & $\begin{array}{l}. \frac{5}{\pi} \\
\sim \\
\sim\end{array}$ & 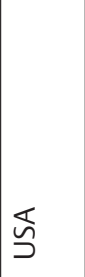 & 芯 \\
\hline 离 & $\Sigma$ & $\Sigma$ & $\supset$ & $\supset$ & $\Sigma$ & $\Sigma$ & $\supset$ & $\supset$ & $\supset$ & $\Sigma$ & $\supset$ & $\supset$ & $\supset$ & $\supset$ & $\Sigma$ & $\supset$ & $\supset$ & $\supset$ & $\Sigma$ & $\supset$ & $\supset$ \\
\hline $\begin{array}{l}\tilde{u} \\
\vdots \\
\vdots \\
\vdots \\
\vdots\end{array}$ & 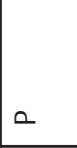 & a & 0 & a & $a$ & $\alpha$ & $a$ & a & $a$ & 0 & 0 & $\simeq$ & a & $\simeq$ & $a$ & $\propto$ & $a$ & 0 & 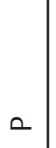 & $\propto$ & $\propto$ \\
\hline 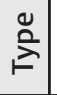 & $\propto$ & $\propto$ & U & $n$ & U & U & $\cup$ & $\cup$ & 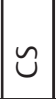 & 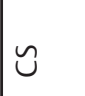 & บ & U & $\cup$ & $\cup$ & 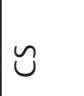 & 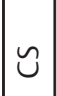 & $\propto$ & $\smile$ & $\mathcal{U}$ & $\cup$ & 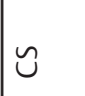 \\
\hline 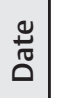 & 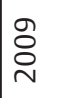 & 임 & $\overline{\text { N }}$ & $\stackrel{\sim}{\sim}$ & $\stackrel{m}{\tilde{D}}$ & $\stackrel{n}{\grave{n}}$ & $\stackrel{m}{\tilde{n}}$ & $\stackrel{m}{\tilde{N}}$ & $\begin{array}{l}m \\
\stackrel{n}{n} \\
\sim\end{array}$ & $\stackrel{m}{\tilde{n}}$ & $\begin{array}{l}m \\
\tilde{n} \\
\end{array}$ & $\begin{array}{l}m \\
\tilde{D} \\
\end{array}$ & $\stackrel{m}{\stackrel{m}{N}}$ & $\stackrel{m}{i}$ & $\stackrel{m}{\stackrel{n}{n}}$ & $\begin{array}{l}+ \\
\stackrel{D}{\sim} \\
\sim\end{array}$ & $\underset{\sim}{\stackrel{D}{\sim}}$ & $\begin{array}{l}\vec{D} \\
\stackrel{2}{D} \\
\sim\end{array}$ & $\underset{⿱ 亠}{D}$ & $\underset{\sim}{\stackrel{D}{\sim}}$ & $\stackrel{\nabla}{\stackrel{D}{\sim}}$ \\
\hline$\frac{0}{\frac{U}{n}}$ & 荵 & 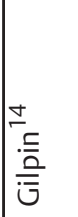 & $\begin{array}{l}\frac{N}{ \pm} \\
0 \\
\frac{1}{1} \\
\frac{1}{0} \\
\tilde{0} \\
n\end{array}$ & 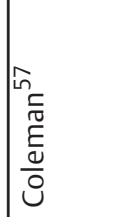 & 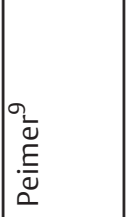 & $\mid$ & 告 & 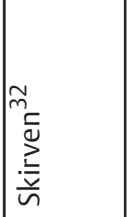 & 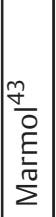 & 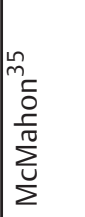 & 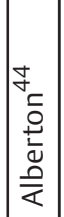 & 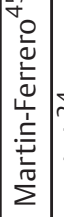 & 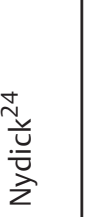 & 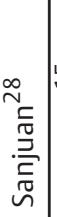 & 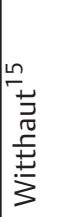 & 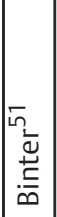 & 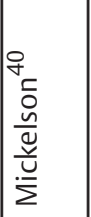 & 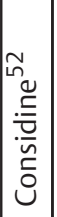 & 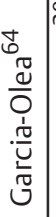 & 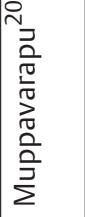 & $\begin{array}{l}0 \\
\cdots \\
\bar{n} \\
0 \\
0 \\
n\end{array}$ \\
\hline
\end{tabular}


Efficacy of Collagenase Clostridium Histolyticum for Dupuytren Disease Sanjuan-Cerveró et al. 75

\begin{tabular}{|c|c|c|c|c|c|c|c|c|c|c|c|c|c|c|c|c|c|c|}
\hline 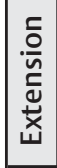 & $\stackrel{\ddagger}{\sim}$ & $\stackrel{\searrow}{\sim}$ & $\approx$ & $\stackrel{\infty}{+}$ & \begin{tabular}{|l}
$\times$ \\
$\infty$ \\
$\infty$ \\
$\stackrel{1}{1}$ \\
$\stackrel{1}{\sim}$ \\
\end{tabular} & $\stackrel{\Xi}{\sim}$ & $\stackrel{\stackrel{\Delta}{\sim}}{ }$ & $\stackrel{\infty}{+}$ & 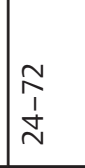 & $\begin{array}{l}0 \\
o \\
1 \\
\stackrel{1}{\sim} \\
\end{array}$ & 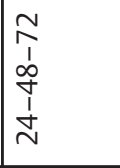 & $\begin{array}{l}\hat{\infty} \\
\stackrel{+}{1} \\
\stackrel{1}{\sim} \\
\stackrel{1}{*}\end{array}$ & $\stackrel{ \pm}{\sim}$ & \begin{tabular}{|l}
$\infty$ \\
$\stackrel{1}{1}$ \\
$\stackrel{1}{\sim}$ \\
\end{tabular} & $\stackrel{\searrow}{\sim}$ & $\stackrel{\searrow}{\sim}$ & $\stackrel{\infty}{+}$ & $\stackrel{\infty}{+}$ \\
\hline \begin{tabular}{|l}
$\stackrel{\Xi}{5}$ \\
$\bar{u}$ \\
$\tilde{J}$ \\
$\Sigma$ \\
$\Sigma$
\end{tabular} & $\sum_{2}$ & 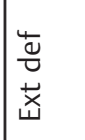 & $\underset{\substack{0 \\
\propto}}{\infty}$ & 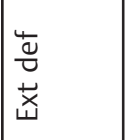 & 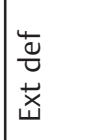 & 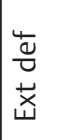 & 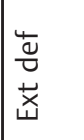 & $\begin{array}{l}\frac{4}{\Delta} \\
\frac{\pi}{x} \\
\ddot{x}\end{array}$ & 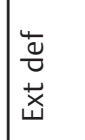 & 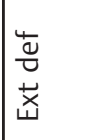 & $\sum_{\propto}$ & 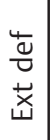 & 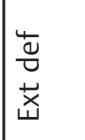 & 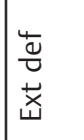 & 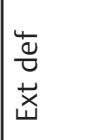 & 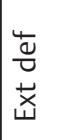 & 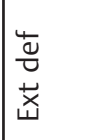 & 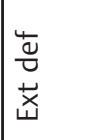 \\
\hline 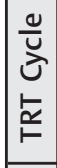 & - & 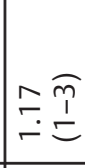 & - & - & $\begin{array}{l}N \\
I\end{array}$ & - & 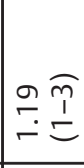 & - & $\underline{\tilde{p}}$ & - & - & $\stackrel{N}{I}$ & $\underline{n}$ & - & $\stackrel{m}{1}$ & $\begin{array}{l}\hat{I} \\
\stackrel{1}{\rightleftharpoons}\end{array}$ & $-\widetilde{T}$ & $\begin{array}{l}\widehat{m} \\
\infty \\
0\end{array}$ \\
\hline $\begin{array}{l}\tilde{u} \\
\text { ̊̊ }\end{array}$ & $\backsim$ & $\backsim$ & $\backsim$ & $\backsim$ & $\gtrless$ & $\gtrless$ & $\backsim$ & $\backsim$ & $\backsim$ & $\backsim$ & ○ै & $\gtrless$ & $\backsim$ & $\backsim$ & $\backsim$ & $\backsim$ & $\backsim$ & $\backsim$ \\
\hline 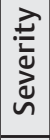 & & & & & & & 总 & 응 & 命 & 总 & 号 & 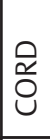 & & 产 & & 号 & 总 & \\
\hline 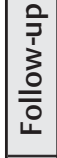 & 8 & ঃ্ & & $\stackrel{\infty}{\circ}$ & $\stackrel{\infty}{\circ}$ & 8 & ৪ & $\stackrel{\stackrel{\sim}{\sigma}}{ }$ & $\underset{\infty}{\ddagger}$ & পু & 8 & $\bar{\sim}$ & 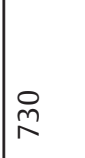 & $\bar{\sim}$ & 㖞 & $\begin{array}{l}\text { Ln } \\
\text { m }\end{array}$ & পீ & $\begin{array}{l}\text { Ln } \\
\text { m }\end{array}$ \\
\hline 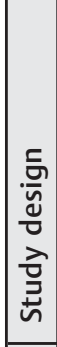 & 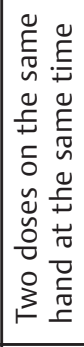 & 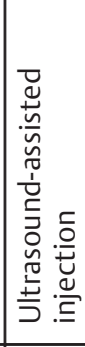 & 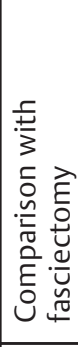 & 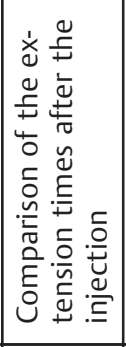 & 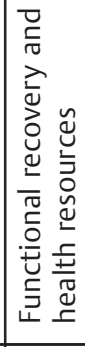 & 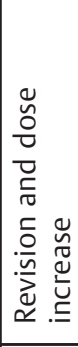 & 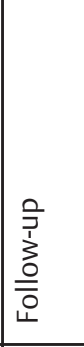 & 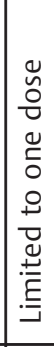 & 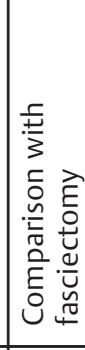 & 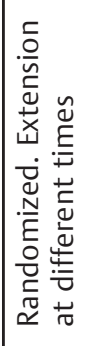 & 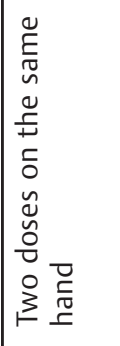 & 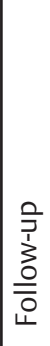 & 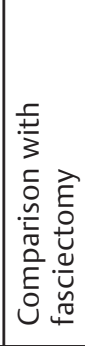 & 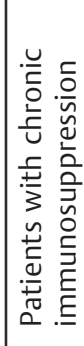 & 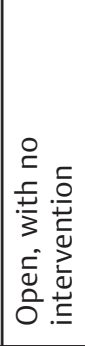 & 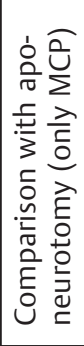 & 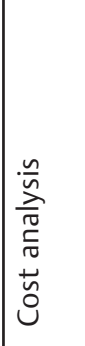 & 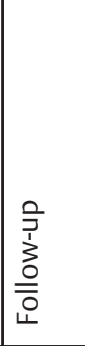 \\
\hline 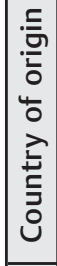 & 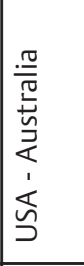 & 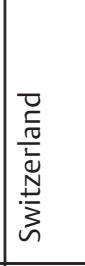 & 兰 & 弚 & 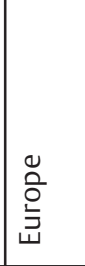 & 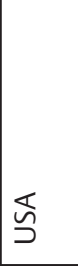 & $\begin{array}{l}\cdot \frac{5}{\pi} \\
\text { nิ }\end{array}$ & 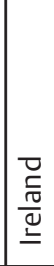 & 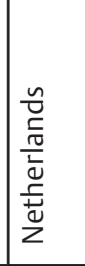 & 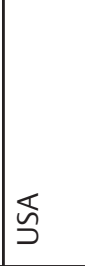 & 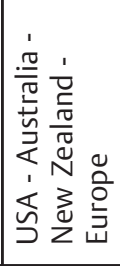 & 离 & 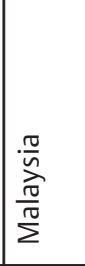 & 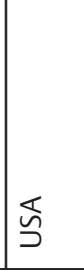 & 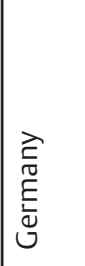 & 离 & 兰 & \begin{tabular}{|l}
$\vec{\pi}$ \\
3 \\
0 \\
0 \\
$z$
\end{tabular} \\
\hline 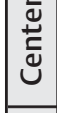 & $\Sigma$ & $\supset$ & $\supset$ & $\supset$ & $\Sigma$ & $\supset$ & $\supset$ & $\supset$ & $\Sigma$ & $\Sigma$ & $\Sigma$ & $\supset$ & $\supset$ & $\Sigma$ & $\Sigma$ & $\supset$ & $\supset$ & $\supset$ \\
\hline 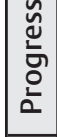 & $\propto$ & $\propto$ & 0 & $\propto$ & a & $\propto$ & 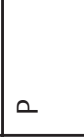 & a & $\propto$ & $a$ & $\circ$ & a & $\propto$ & $\propto$ & $a$ & $a$ & $a$ & $a$ \\
\hline 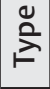 & $\cup$ & $\tilde{U}$ & $\cup$ & ป & 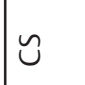 & 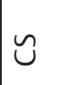 & U & U & $\cup$ & U & U & U & $\cup$ & 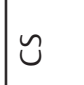 & 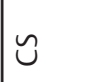 & $\cup$ & U & U \\
\hline 苋 & $\frac{\nabla}{\grave{N}}$ & $\frac{\text { d }}{\stackrel{N}{N}}$ & $\stackrel{+}{\stackrel{D}{\sim}}$ & $\frac{\pi}{\stackrel{N}{2}}$ & $\frac{\text { d }}{\stackrel{N}{N}}$ & $\stackrel{+}{\stackrel{\sim}{n}}$ & ำ & $\stackrel{n}{\stackrel{n}{\sim}}$ & $\stackrel{n}{\stackrel{n}{\sim}}$ & $\stackrel{n}{\stackrel{n}{N}}$ & ำ & $\stackrel{n}{\stackrel{n}{\sim}}$ & 㫯 & 站 & 站 & 号 & 号 & 号 \\
\hline 誉 & 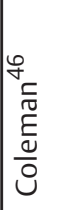 & 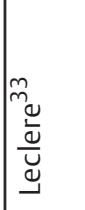 & 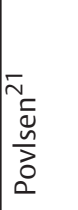 & 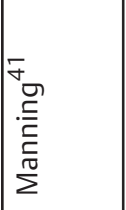 & 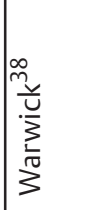 & 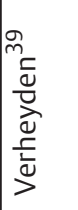 & 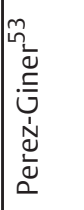 & 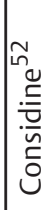 & $\begin{array}{l}\tilde{N}_{z} \\
\frac{\partial}{N}\end{array}$ & 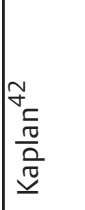 & 传 & 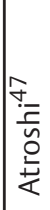 & 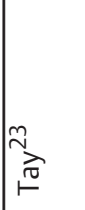 & 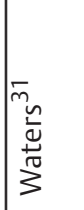 & 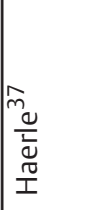 & 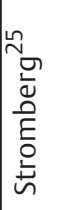 & 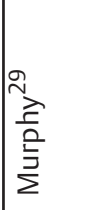 & 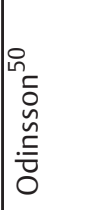 \\
\hline
\end{tabular}




\begin{tabular}{|c|c|c|c|c|c|c|c|c|c|c|c|c|}
\hline 芉 & $\stackrel{\triangleright}{\sim}$ & $\stackrel{\Xi}{\sim}$ & & 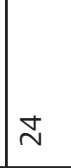 & 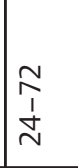 & $\stackrel{\searrow}{\sim}$ & \begin{tabular}{l}
$\infty$ \\
$\stackrel{1}{1}$ \\
\multirow{2}{\pm}{} \\
\end{tabular} & $\stackrel{\Xi}{\sim}$ & $\stackrel{\Xi}{\sim}$ & $\stackrel{\searrow}{\sim}$ & $\stackrel{\sim}{\sim}$ & 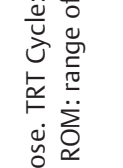 \\
\hline 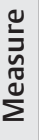 & $\begin{array}{l}\vec{y} \\
\frac{d}{0} \\
\vec{x}\end{array}$ & $\sum_{\substack{1 \\
\propto}}$ & 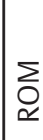 & 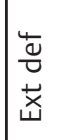 & 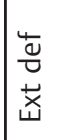 & $\sum_{\substack{2 \\
\propto}}$ & 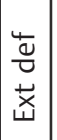 & 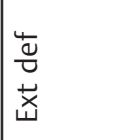 & $\begin{array}{l}\frac{4}{\tilde{y}} \\
\vec{x} \\
\vec{x}\end{array}$ & 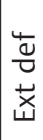 & 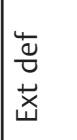 & 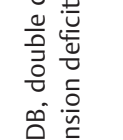 \\
\hline 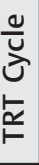 & $\stackrel{m}{r}$ & $\stackrel{\widehat{m}}{\stackrel{1}{=}}$ & $\stackrel{m}{\stackrel{m}{c}}$ & $-\overline{1}$ & - & 꼴 & - & - & $\stackrel{\widehat{m}}{\stackrel{1}{=}}$ & $\bar{m}$ & - & 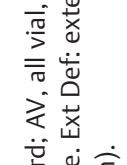 \\
\hline ڤั̆ & $\sim$ & $\sim$ & $\backsim$ & $\backsim$ & $\sim$ & $\backsim$ & $\gtreqless$ & $\gtreqless$ & $n$ & $\backsim$ & $\backsim$ & 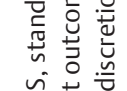 \\
\hline : & 产 & 产 & 产 & 产 & & 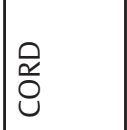 & & 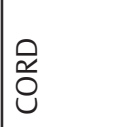 & \% & & & 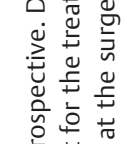 \\
\hline $\begin{array}{l}\frac{0}{7} \\
3_{0}^{1} \\
\text { ō } \\
\end{array}$ & $\begin{array}{l}n \\
0 \\
m\end{array}$ & $\begin{array}{l}n \\
0 \\
m \\
\end{array}$ & প & $\stackrel{\infty}{\infty}$ & 望 & 知 & 间 & in & $\stackrel{2}{0}$ & 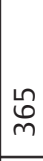 & $\stackrel{p}{r}$ & 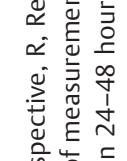 \\
\hline 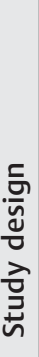 & 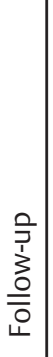 & ठ̃ & 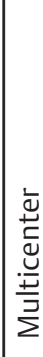 & $\begin{array}{l}\frac{0}{7} \\
3 \\
\text { 3. } \\
\overline{0} \\
1\end{array}$ & 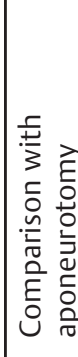 & 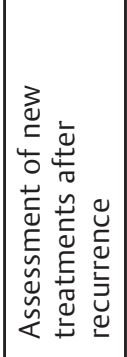 & $\begin{array}{c} \\
0 \\
0 \\
3 \\
0 \\
0 \\
0\end{array}$ & 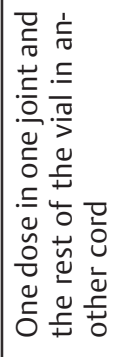 & 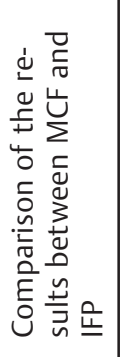 & 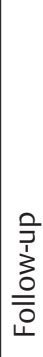 & 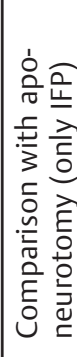 & 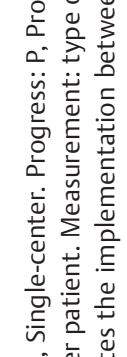 \\
\hline 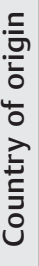 & 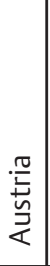 & \begin{tabular}{|l} 
踣 \\
蒠
\end{tabular} & $\frac{\underline{E}}{\frac{5}{\bar{V}}}$ & 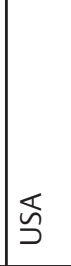 & 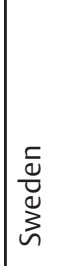 & 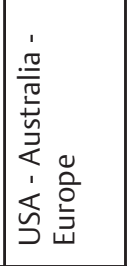 & 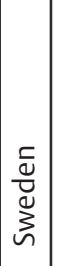 & 芩 & 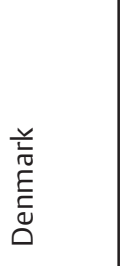 & 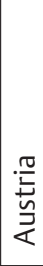 & 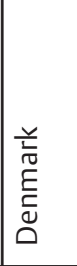 & 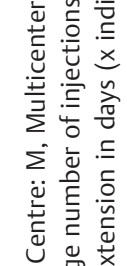 \\
\hline ن & $\supset$ & $\Sigma$ & $\Sigma$ & $\supset$ & $\Sigma$ & $\Sigma$ & $\supset$ & $\backsim$ & $\backsim$ & $\backsim$ & $\backsim$ & 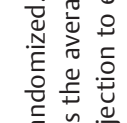 \\
\hline 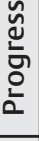 & $\simeq$ & 0 & 2 & $\propto$ & $a$ & $a$ & 0 & $\simeq$ & $a$ & $a$ & $a$ & 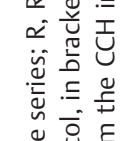 \\
\hline$\stackrel{\Xi}{ٍ}$ & 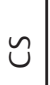 & 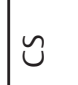 & 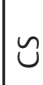 & U & $u$ & U & $\mathcal{U}$ & U & $\mho$ & $\tilde{U}$ & $\cup$ & $\ddot{U}$ \\
\hline 䒕 & $\begin{array}{l}0 \\
\stackrel{N}{N}\end{array}$ & i & 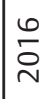 & i & 吕 & $\stackrel{i}{\grave{N}}$ & $\begin{array}{l}\hat{n} \\
\tilde{D}\end{array}$ & 立 & $\stackrel{\hat{D}}{\sim}$ & $\hat{\tilde{N}}$ & 홍 & $\begin{array}{l}0 \\
\overline{0} \\
\end{array}$ \\
\hline 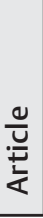 & 妾 & 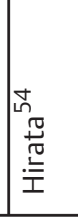 & | & 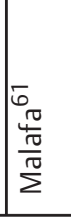 & 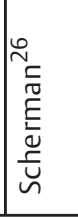 & 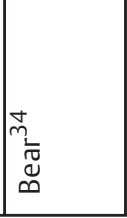 & 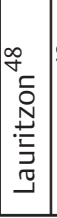 & 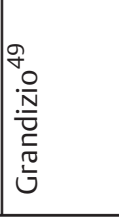 & 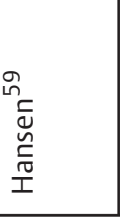 & $\begin{array}{l}\frac{8}{0} \\
\frac{\bar{\omega}}{\bar{Q}} \\
\underline{\underline{y}}\end{array}$ & $\begin{array}{l}n \\
0 \\
\text { v } \\
\end{array}$ & 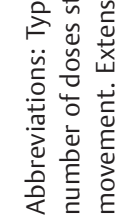 \\
\hline
\end{tabular}


Efficacy of Collagenase Clostridium Histolyticum for Dupuytren Disease Sanjuan-Cerveró et al. 77

Table 2 Demographic features of the studies analyzed

\begin{tabular}{|c|c|c|c|c|c|c|}
\hline Article & Date & $\mathrm{N}^{\circ} \mathrm{CCH}$ & Mean age & Male (\%) & Previous TRT & Fam His \\
\hline Hurst $^{6}$ & 2009 & 203 & 62.7 & 171 & 79 & 85 \\
\hline Gilpin $^{14}$ & 2010 & 66 & 63.8 & 39 & 28 & 22 \\
\hline Spanholtz $^{18}$ & 2011 & 8 & 62.5 & 6 & & \\
\hline Coleman $^{57}$ & 2012 & 12 & 63.7 & 11 & 5 & 6 \\
\hline Peimer $^{9}$ & 2013 & 643 & 66 & 542 & & 278 \\
\hline Peimer ${ }^{65}$ & 2013 & 463 & 65.5 & 342 & & \\
\hline Vollbach $^{19}$ & 2013 & 14 & & & & \\
\hline Skirven $^{32}$ & 2013 & 21 & 63 & 19 & 12 & \\
\hline Marmol $^{43}$ & 2013 & 15 & 64 & & & \\
\hline McMahon $^{35}$ & 2013 & 64 & $\mathrm{~N} / \mathrm{A}$ & 31 & & \\
\hline Alberton $^{44}$ & 2013 & 40 & 66 & 36 & & \\
\hline Martin-Ferrero $^{45}$ & 2013 & 35 & 68.1 & 35 & 3 & \\
\hline Nydick $^{24}$ & 2013 & 29 & 67 & 25 & & \\
\hline Sanjuan 28 & 2013 & 91 & 65.1 & 38 & & \\
\hline Witthaut $^{15}$ & 2013 & 587 & 63.7 & 498 & 308 & 245 \\
\hline Binter $^{51}$ & 2014 & 37 & 66 & 32 & 0 & \\
\hline Mickelson $^{40}$ & 2014 & 43 & 64.5 & 35 & 10 & 19 \\
\hline Considine $^{52}$ & 2014 & 10 & 66 & 10 & 2 & \\
\hline Garcia-Olea $^{64}$ & 2014 & 148 & 64 & 140 & 24 & 44 \\
\hline Muppavarapu $^{20}$ & 2014 & 73 & 64 & 61 & 24 & \\
\hline Sood $^{30}$ & 2014 & 16 & 69.9 & 16 & & 2 \\
\hline Coleman $^{46}$ & 2014 & 60 & 64 & 51 & 26 & 25 \\
\hline Leclere $^{33}$ & 2014 & 33 & 64.4 & 28 & & \\
\hline Povlsen $^{21}$ & 2014 & 10 & & & & \\
\hline Manning $^{41}$ & 2014 & 45 & 63 & 33 & 8 & \\
\hline Warwick $^{38}$ & 2014 & 144 & $\mathrm{~N} / \mathrm{A}$ & 119 & & \\
\hline Verheyden ${ }^{39}$ & 2014 & 40 & 66 & 38 & & \\
\hline Perez-Giner ${ }^{53}$ & 2015 & 10 & 65.6 & 7 & & \\
\hline Considine $^{52}$ & 2015 & 104 & 61 & 83 & 27 & 56 \\
\hline Zhou $^{22}$ & 2015 & 37 & 65.5 & 34 & & \\
\hline Kaplan $^{42}$ & 2015 & 714 & 64 & 616 & 376 & 337 \\
\hline Gaston $^{17}$ & 2015 & 164 & 70 & 134 & 23 & \\
\hline Atroshi $^{47}$ & 2015 & 29 & 65 & 13 & & \\
\hline Tay $^{23}$ & 2015 & 237 & 64 & & & \\
\hline Waters $^{31}$ & 2015 & 8 & 66 & & & \\
\hline Haerle $^{37}$ & 2015 & 86 & 65.1 & 69 & & 28 \\
\hline Stromberg $^{25}$ & 2016 & 69 & 66 & 56 & 0 & 34 \\
\hline Murphy $^{29}$ & 2016 & 20 & 64.8 & 20 & & 11 \\
\hline Odinsson $^{50}$ & 2016 & 77 & 69 & 66 & & \\
\hline Arora $^{58}$ & 2016 & 120 & 62 & & & \\
\hline Hirata ${ }^{54}$ & 2016 & 77 & 68 & 70 & 35 & \\
\hline Verstreken $^{55}$ & 2016 & 104 & 64.4 & 85 & 85 & 47 \\
\hline Malafa 61 & 2016 & 36 & 65.1 & 33 & & \\
\hline
\end{tabular}


Table 2 (Continued)

\begin{tabular}{|c|c|c|c|c|c|c|}
\hline Article & Date & $\mathrm{N}^{\circ} \mathrm{CCH}$ & Mean age & Male (\%) & Previous TRT & Fam His \\
\hline Scherman $^{26}$ & 2016 & 56 & & 36 & & \\
\hline $\mathrm{Bear}^{34}$ & 2017 & 52 & 66.5 & 50 & 52 & \\
\hline Lauritzon $^{48}$ & 2017 & 48 & 68 & 38 & 6 & \\
\hline Grandizio $^{49}$ & 2017 & 34 & 65 & 23 & & 5 \\
\hline Hansen $^{59}$ & 2017 & 212 & 66 & 195 & 0 & \\
\hline Keller $^{60}$ & 2017 & 120 & 62 & 107 & & \\
\hline Skov 27 & 2017 & 29 & 62 & 45 & & \\
\hline
\end{tabular}

Abbreviations: Fam His, Number of patients in the series with a family history of DD; $\mathrm{N}^{\circ} \mathrm{CCH}$, Number of patients treated with $\mathrm{CCH}$ in the study; Previous TRT, Number of patients in the series who have received prior treatment for DD on the finger treated with CCH (any).

the studies by Gilpin ${ }^{14}(1.5)$ and $\operatorname{Sood}^{30}(1.6)$ being the ones that used more than one vial per joint. The extension time varied among the studies, ranging from 24 hours (predominantly) up to 7 days in a clinical trial by Mickelson. ${ }^{40}$

The systematic implementation of a physiotherapy protocol after $\mathrm{CCH}$ treatment is another controversial point regarding the outcomes. Thirteen articles indicate that patients were systematically referred to an established physiotherapy protocol or that monitoring was performed by specialist physiotherapists, ${ }^{20,25,29,32,34,35,40,41,44,48,51-53}$ four specify that patients did not receive physical therapy, ${ }^{6,22,49,54}$ two have indicated it only if necessary, ${ }^{46,55}$ and the rest did not indicate whether or not a physiotherapy protocol was included in the treatment protocol.

Using night-time splints is recommended for a period of 3 months in the product fact sheet. ${ }^{56}$ The bibliography is variable in this regard. Twenty-seven articles refer to the systematic use of this device, $6,14,15,17,20,22,25,26,30,33,35,36,38-41,44,46-49,51,54,57-60$ and a multicenter study reports that only one of the centers used it without performing a statistical analysis of what this measurement could indicate. ${ }^{61}$

\section{Clinical Findings}

The studies analyzed indicate a timeline regarding the progression time. In spite of not having a formal indication as to the progression time, the studies mark a before and an after with a timeline of less or more than 1 year. Studies with a course of less than 1 year set out to assess the clinical effectiveness of the treatment or some of its modifications (if the treatment actually works by reducing contracture in DD), and the studies that took 1 year or longer try to assess the effectiveness of the treatment (if the achieved effect is maintained over time). To calculate the clinical outcomes ( Appendix A), data from studies that lasted less than 1 year ( - Table 3) have been taken into account together with the figures from the intermediate results of the studies that took 1 year or longer ( $\mathbf{- T a b l e} \mathbf{4})$.

\section{Studies that Cover Less than 1 Year of Progress} The mean follow-up time was 104 (SD: 75) days. The analysis included a total of 4,666 joints (average of 194.41 joints per study), (2,467 MCPs (average of 129.84) and 1,516 PIPs (average of 75.80$)$ ). The primary end point objective has been achieved in $48.9 \%$ of the treated joints $(69.77 \%$ of the MCPs and $30.14 \%$ of the PIPs). The mean correction for all joints treated was 45.5 (SD: 19.18 ) degrees; 40.8 degrees for MCPs (SD: 10.12) and 35.6 for PIPs (SD: 13.23). The proportional correction of the joints was at $72.9 \%$ (SD: 14.43) overall, (83.9\% for the MCP [SD: 12.58] and 64.2 for the PIP [SD: 16.35]).

\section{Studies that Cover More than 1 Year of Progress}

The mean follow-up time was 467 days (SD: 196.36). The analysis included a total of 2,870 joints (average of 138.66 joints per study), (1,459 MCPs [average of 97.26] and 842 PIPs [average of 56.13]). The primary end point objective has been achieved in $57.5 \%$ of the treated joints ( $68.9 \%$ of the MCPs and $43.3 \%$ of the PIPs). The mean correction for all joints treated was 37.6 degrees (SD: 10.93), (37.3 degrees for the MCP [SD: 9.98] and 23.7 for the PIP [SD: 16.33]. The proportional correction of joints was at $87.3 \%$ (SD: 10.96) overall, (90.3\% for the MCP [SD: 6.94] and 75\% for the PIP [SD: 13.54]).

\section{Recurrences}

Apart from the CORDLESS studies, ${ }^{9,10}$ few studies have collected the incidence of recurrences in their results. ${ }^{19,30,34,35,37,48,58}$ The rate of recurrence during the follow-up period ranged from $1.7 \%$ in 1 year $^{37}$ to $59 \%$ in the series by Sood $^{30}$ within the same period, but with its own criteria of recurrence. Upon eliminating these two values, this rate ranged between $7-28 \%$. All the articles that mentioned the rate of recurrence had a follow-up between 6 and 18 months. We should keep in mind that the series by Bear ${ }^{34}$ had a $28 \%$ recurrence rate in patients previously treated with $\mathrm{CCH}$ after 1 year of follow-up.

The CORDLESS studies were designed as observational clinical studies for assessing the rate of recurrence over time. These follow-ups were published at $3^{9}$ and 5 years. ${ }^{10}$ The recurrence rate was calculated based on the secondary end point (at least $50 \%$ of correction since the initial contracture), demonstrating a recurrence rate of $38 \%$ (28\% for the MCP and $58 \%$ for the PIP) at 3 years, and $48 \%$ (39\% for the MCP and $65 \%$ for the PIP) at 5 years. Peimer indicates that $75 \%$ of the 


\begin{tabular}{|c|c|c|c|c|c|c|c|c|c|c|c|c|c|c|c|c|c|c|}
\hline $\begin{array}{l}\text { 릉 } \\
\text { 웅 }\end{array}$ & ఫ̊ & $\stackrel{\stackrel{0}{\cdot}}{i n}$ & & $\stackrel{m}{\stackrel{m}{ }}$ & 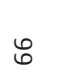 & $\infty$ & Бे & & & กิ & นั่ & $\stackrel{\infty}{+}$ & & $\mathscr{\theta}$ & in & & $\stackrel{2}{\gtrless}$ & \\
\hline 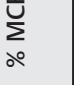 & $\infty$ & 旾 & & $\stackrel{\infty}{\infty}$ & $\bar{\infty}$ & & $\stackrel{8}{\circ}$ & & & $\stackrel{\infty}{\infty}$ & $\underset{\infty}{ \pm}$ & 占 & & $\infty$ & ठ & & $\infty$ & \\
\hline ○ீ & g & $\stackrel{\text { n̊ }}{\stackrel{2}{n}}$ & & $\hat{i}$ & $\stackrel{2 n}{\wedge}$ & & $\stackrel{\infty}{\circ}$ & & & 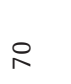 & $\stackrel{b}{\stackrel{i}{N}}$ & $\begin{array}{l}\text { ñ } \\
\text { g }\end{array}$ & & & in & & & \\
\hline 壳 & 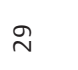 & $\approx$ & $\tilde{m}$ & $\bar{m}$ & $\stackrel{J}{m}$ & g & & 6 & $\stackrel{ \pm}{\sim}$ & $\tilde{m}$ & $\stackrel{\llcorner}{\sim}$ & $\stackrel{\infty}{+}$ & $\stackrel{m}{m}$ & $\lesssim$ & $\stackrel{t}{\wedge}$ & $m$ & $\bar{m}$ & m \\
\hline ¿ & $\bar{\nabla}$ & $\mathscr{F}$ & $m$ & શิ & $\stackrel{\infty}{m}$ & & f & in & $\stackrel{\text { m }}{\text { s. }}$ & $\mathscr{F}$ & 늣 & ஜே & in & $\approx$ & g̊ & $q$ & $m$ & 它 \\
\hline 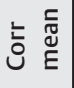 & $\hat{\dot{m}}$ & 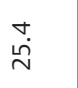 & $m$ & iे & & & $\exists$ & 8 & & & & & & ถิ & & & & \\
\hline 은 & & & & & & & & $\widehat{\imath}$ & & & & & & & $\stackrel{\infty}{\sim}$ & \& & $\stackrel{m}{m}$ & \\
\hline Ü & & & & & & & & $\bar{\gamma}$ & & & & & & & g & g & $\stackrel{\infty}{m}$ & \\
\hline 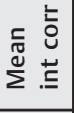 & & & & & & & & 8 & & & & & & & & & & \\
\hline 兽 & ₹ $\widehat{O}$ & $\infty \mathbb{\mathbb { N }}$ & & & $0 \stackrel{\widehat{\Phi}}{\mathbb{d}}$ & & & & & & $+\overline{\grave{d}}$ & $\simeq \widetilde{\tilde{n}}$ & & & & & & \\
\hline$\sum_{\breve{U}}^{\stackrel{U}{u}}$ & 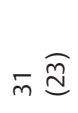 & $\wedge \stackrel{\sqrt{\Omega}}{0}$ & & & $\sigma \stackrel{\sqrt[\Omega]{\varrho}}{=}$ & & & & & & $\overline{0} \stackrel{\widehat{0}}{=}$ & ○宇 & & & & & & \\
\hline 亗 & 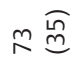 & $\stackrel{\sim}{\sim} \stackrel{\widehat{n}}{\mathscr{n}}$ & & & $\stackrel{\Perp}{\beth} \underset{\Omega}{=}$ & & & & & & $\stackrel{n}{\stackrel{(a}{=}}$ & $\approx \stackrel{\widehat{D}}{+}$ & & & & & & \\
\hline $\begin{array}{l}\stackrel{\underline{\underline{u}}}{\underline{\underline{u}}} \\
\underline{\underline{u}}\end{array}$ & $\stackrel{\infty}{\sim} \stackrel{\widehat{Q}}{ \pm}$ & $\wedge \stackrel{\widehat{\infty}}{\stackrel{d}{(}}$ & & & $\sigma \stackrel{\mathscr{Q}}{d}$ & & $\wedge \stackrel{\widehat{\triangleright}}{\varrho}$ & & & & $\stackrel{\infty}{\simeq} \stackrel{0}{0}$ & $\infty \stackrel{\widehat{\infty}}{\stackrel{0}{n}}$ & & $\stackrel{\sim}{\underline{m}}$ & & & $m \widetilde{\widetilde{I}}$ & \\
\hline $\begin{array}{l}\sum_{0}^{\breve{U}} \\
\bar{\Delta}\end{array}$ & $\therefore \stackrel{0}{\subseteq}$ & m $\stackrel{\widehat{\Omega}}{\widehat{C}}$ & & & $\underset{\sim}{\widetilde{N}}$ & & $\underset{\sim}{\stackrel{\partial}{\circ}}$ & & & & 甪高 & 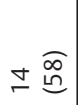 & & in $\stackrel{0}{=}$ & $\mathscr{m} \widetilde{\sigma}$ & & $\approx \widehat{\Sigma}$ & \\
\hline 屵 & 官点 & 。 & & & 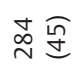 & & $\bar{m} \stackrel{\widehat{o}}{=}$ & ๙ & & & 可高 & 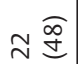 & & $\therefore \stackrel{0}{0}$ & & & & \\
\hline $\begin{array}{l}\frac{2}{0} \\
\frac{1}{z}\end{array}$ & 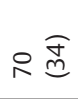 & 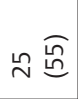 & & $\pm \widehat{\infty}$ & $\bar{\sim} \underset{\sim}{0}$ & $\approx \stackrel{\circ}{\circ}$ & 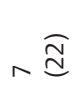 & 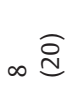 & $n \stackrel{\widehat{\sigma}}{\Xi}$ & $\bar{m}$ 官 & 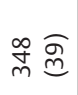 & $\bar{\sim}$ & & 㚈 $\underset{\tilde{m}}{\widetilde{m}}$ & $\infty \stackrel{\widehat{\varrho}}{=}$ & & $i_{m}^{\frac{\alpha}{z}}$ & \\
\hline $\begin{array}{l}\sum_{2}^{U} \\
\vdots \\
z\end{array}$ & $\stackrel{m}{m} \stackrel{\widehat{\Omega}}{0}$ & $\stackrel{\widetilde{J}}{\mathrm{~J}}$ & & $\approx \widehat{\bar{\sigma}}$ & $\stackrel{\infty}{\stackrel{m}{m} \widehat{\underline{\theta}}}$ & & $\underset{\sim}{\mathbb{I}}$ & $\approx \widetilde{\mathscr{\infty}}$ & 士可 & 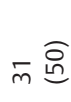 & 向 $\widehat{o}$ & $\stackrel{\sim}{\widetilde{\tilde{m}}}$ & & 능 & $\approx \underset{\infty}{\stackrel{f}{\infty}}$ & & 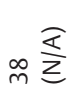 & \\
\hline 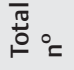 & $\stackrel{\sim}{\sim}$ & ஜே & $\stackrel{\bullet}{\circ}$ & $\stackrel{m}{m}$ & శ్రి & $\stackrel{\sim}{\sim}$ & $\bar{m}$ & q & 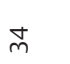 & $\widetilde{6}$ & $\underset{\infty}{\Omega}$ & ஜq & $\stackrel{m}{\sim}$ & $\stackrel{\text { I }}{2}$ & in & $\overline{\tilde{n}}$ & & $m$ \\
\hline 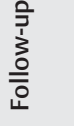 & ৪ & ๑ & $\stackrel{\nabla}{\tau}$ & $\stackrel{尺}{\sim}$ & $\stackrel{一}{\text { m }}$ & 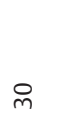 & $\stackrel{\circ}{m}$ & $\stackrel{\infty}{-}$ & $\stackrel{\infty}{-}$ & $\stackrel{\infty}{-}$ & $\stackrel{尺}{N}$ & m & ๒ & 8 & $\infty$ & $\stackrel{\infty}{-}$ & 8 & ৪) \\
\hline 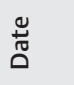 & ڤे & 을 & $\bar{\sim}$ & $\stackrel{\sim}{\stackrel{N}{\sim}}$ & $\stackrel{m}{\stackrel{n}{N}}$ & 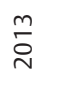 & $\stackrel{m}{\stackrel{m}{\sim}}$ & $\stackrel{m}{\stackrel{m}{\sim}}$ & $\stackrel{m}{\stackrel{m}{\sim}}$ & $\stackrel{m}{\stackrel{m}{\sim}}$ & $\stackrel{m}{\stackrel{m}{n}}$ & $\stackrel{+}{\stackrel{N}{N}}$ & $\stackrel{\vec{D}}{\stackrel{N}{\sim}}$ & $\stackrel{\nabla}{\stackrel{\sim}{N}}$ & $\stackrel{\nabla}{\stackrel{N}{N}}$ & $\stackrel{\vec{\sigma}}{\vec{\sim}}$ & $\stackrel{+}{\stackrel{N}{N}}$ & 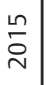 \\
\hline 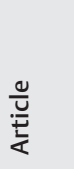 & 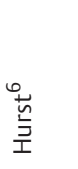 & 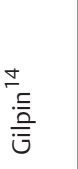 & 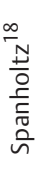 & 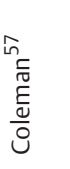 & 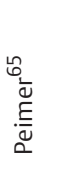 & 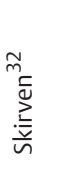 & 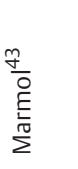 & 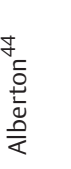 & 离 & 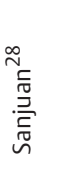 & 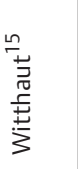 & 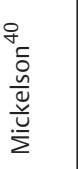 & 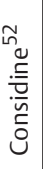 & 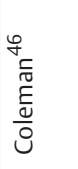 & 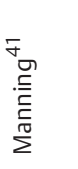 & 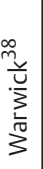 & 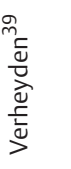 & 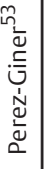 \\
\hline
\end{tabular}




\begin{tabular}{|c|c|c|c|c|c|c|c|c|c|c|c|}
\hline$\frac{\text { 妾 }}{\circ o}$ & & & & 8 & & $\tilde{m}$ & & & & $\infty$ & 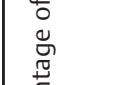 \\
\hline 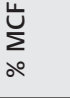 & & & & $\infty$ & & $\infty$ & & & & นn & 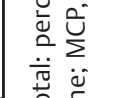 \\
\hline ஃ゚ & & & & i & & & & $\overline{\dot{0}}$ & & & 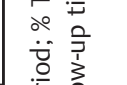 \\
\hline 记 呈 & & $\stackrel{\Delta}{\sim}$ & & & $\stackrel{\infty}{\sim}$ & $\stackrel{\mathscr{1}}{2}$ & $q$ & & $\stackrel{\infty}{m}$ & $\stackrel{g}{m}$ & 9 \\
\hline ¿ & & $m$ & 年 & & $\stackrel{g}{m}$ & $m$ & $\stackrel{\infty}{m}$ & & F & 年 & $\begin{array}{l}\text { 응 } \\
\text { 응 } \\
0\end{array}$ \\
\hline 气 & & & & 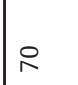 & 望 & & $m$ & \& & q & $\infty$ & 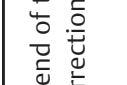 \\
\hline$\underline{\underline{\underline{B}}}$ & & & & & & & & & & & \\
\hline Uั & & & & & & & & & & & 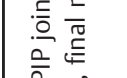 \\
\hline 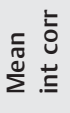 & & & & 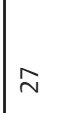 & & & & & & & 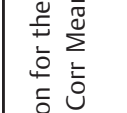 \\
\hline 㦈 & & $\pm \widehat{\mathcal{I}}$ & & $\widehat{\widetilde{N}} \widehat{\mathcal{I}}$ & & & & n $\cong$ & & & 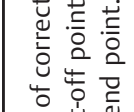 \\
\hline$\sum_{\breve{U}}^{\stackrel{U}{u}}$ & & $\stackrel{\sqrt{n}}{\stackrel{5}{S}}$ & & $\bar{\sim} \stackrel{\mathcal{N}}{\mathcal{I}}$ & & & & $\tilde{\sim} \overline{\bar{m}}$ & & & 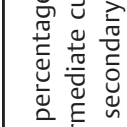 \\
\hline 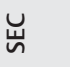 & & 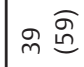 & & 舲 $\overline{\bar{n}}$ & & & & $\stackrel{\infty}{\stackrel{(n}{\sim}}$ & & & \\
\hline $\begin{array}{l}\text { 는 } \\
\text { 产 }\end{array}$ & in & & & $\stackrel{\infty}{\stackrel{\infty}{\sim} \stackrel{\infty}{\simeq}}$ & & & & $\pm \stackrel{\overparen{\Omega}}{n}$ & & $\simeq \frac{\bar{s}}{\Sigma}$ & 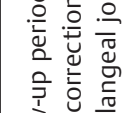 \\
\hline $\begin{array}{l}\sum_{u}^{u} \\
\text { ù }\end{array}$ & $\simeq \stackrel{\widehat{\circ}}{=}$ & & $2 \stackrel{\frac{\pi}{3}}{2}$ & 周委 & & & & $\stackrel{\infty}{\stackrel{\infty}{\complement}}$ & & i $\frac{\frac{\pi}{3}}{3}$ & $E$ \\
\hline 㟔 & $m \stackrel{\widehat{\Xi}}{c}$ & & & $\hat{⿱} \vec{\gamma} \stackrel{0}{0}$ & & & & $\approx$ F & $\stackrel{0}{0} \stackrel{0}{\Omega}$ & $\approx \widetilde{A}$ & 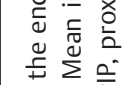 \\
\hline $\begin{array}{l}\frac{i}{\underline{\underline{x}}} \\
\frac{1}{z}\end{array}$ & in $\stackrel{\widehat{\infty}}{\stackrel{\infty}{n}}$ & 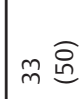 & & 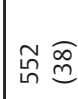 & & $\infty \stackrel{\widehat{\Xi}}{\mathbb{I}}$ & $0 \stackrel{0}{9}$ & q & $\wedge \stackrel{\Im}{\Xi}$ & & 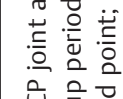 \\
\hline 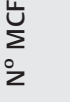 & $\simeq \widehat{\widetilde{a}}$ & m $\overline{\text { ing }}$ & & 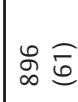 & & $\bar{\sim} \widehat{\mathbb{N}}$ & $\wedge \widehat{\hat{n}}$ & $\triangleright \delta$ & q & & $g$ \\
\hline 胥 。 & & & 8 & $\underset{⿱}{\stackrel{S}{+}}$ & 吕 & $\stackrel{\mathscr{N}}{\sim}$ & $m$ & $\mp$ & F & $\dot{m}$ & 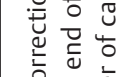 \\
\hline 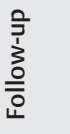 & & 年 & 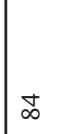 & ஃ & 8 & $\bar{\sim}$ & $\bar{\sim}$ & ৪ & $\stackrel{\infty}{-}$ & $\stackrel{\circ}{\mathrm{m}}$ & 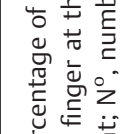 \\
\hline D̃ & & $\frac{n}{2}$ & 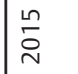 & $\stackrel{n}{2}$ & $\frac{n}{2}$ & 知 & $\frac{n}{2}$ & $\frac{0}{2}$ & 号 & $\hat{n}$ & 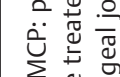 \\
\hline 莺 & & 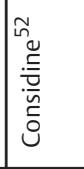 & $\begin{array}{l}\tilde{N} \\
\bar{\partial} \\
\frac{\bar{N}}{N}\end{array}$ & 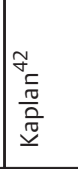 & 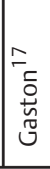 & 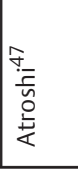 & 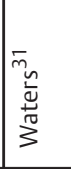 & 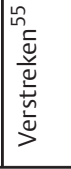 & 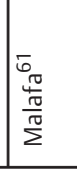 & 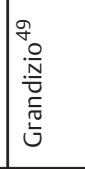 & 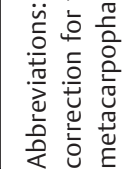 \\
\hline
\end{tabular}




\begin{tabular}{|c|c|c|c|c|c|c|c|c|c|c|c|c|c|c|c|c|c|c|}
\hline$\frac{\text { 产 }}{20}$ & & & & & & & & 占 & & б & & & & & & $\cong$ & & 8 \\
\hline 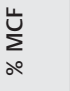 & & & & & & & & $\underset{\infty}{m}$ & & à & & & & & & à & & $\infty$ \\
\hline 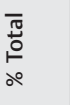 & & & & & & & & & & ட & & & & & & б & $\stackrel{n}{N}$ & \\
\hline 产 & $\stackrel{\llcorner}{\llcorner}$ & & 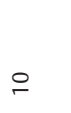 & $\stackrel{\infty}{\llcorner}$ & & $\tilde{\sim}$ & $\hat{m}$ & $\stackrel{\text { N }}{ }$ & $\bar{N}$ & 吕 & $\infty$ & & 6 & $\stackrel{0}{\circ}$ & $\stackrel{\rho}{m}$ & $\stackrel{\llcorner}{m}$ & $\tau$ & $\bar{m}$ \\
\hline ¿ & $\stackrel{\infty}{\sim}$ & & $\approx$ & 8 & & $\stackrel{m}{m}$ & $\bar{N}$ & $\dot{m}$ & $\stackrel{\infty}{\sim}$ & \& & $\bar{m}$ & f & $\stackrel{m}{m}$ & $\stackrel{\infty}{\sim}$ & $\stackrel{\infty}{\sim}$ & $\tilde{F}$ & in & $m$ \\
\hline 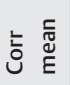 & $\bar{N}$ & $\bar{m}$ & $m$ & & & & $\stackrel{\infty}{\sim}$ & & & & & f & & & & q & 多 & $\tilde{m}$ \\
\hline 을 & & & $\stackrel{\sim}{\sim}$ & 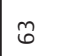 & & in & & & $\bar{m}$ & & $\stackrel{\sim}{\sim}$ & & $\stackrel{\infty}{\rightleftharpoons}$ & $\tilde{\sim}$ & & & $m$ & \\
\hline ¿ّ & & & f & ڤ̊n & & $\stackrel{\infty}{m}$ & & & $m$ & & $\stackrel{\infty}{\sim}$ & 요 & ஜ & $\approx$ & & & 缉 & \\
\hline 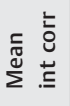 & & $m$ & $\bar{\gamma}$ & & & & & & & & & in & & & & & $\stackrel{\infty}{+}$ & \\
\hline $\begin{array}{l}\text { 咅 } \\
\text { 㞾 }\end{array}$ & 守 $\underset{\sim}{f}$ & & & & & & & $\sigma \stackrel{\bar{\partial}}{=}$ & & n $\widehat{\infty}$ & & & & & & $m \stackrel{\widehat{\varrho}}{=}$ & & \\
\hline 岀 & $\hat{\sim} \widetilde{\widetilde{d}}$ & & & & & & & 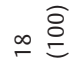 & & $m \widehat{n}$ & & & & & & $\circ \widehat{\varrho}$ & & \\
\hline 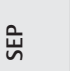 & $\bar{m} \underset{\mathrm{d}}{\widehat{\Xi}}$ & & & & & & & $\lesssim \stackrel{\widehat{\partial}}{=}$ & & $\infty \widehat{\mathbb{a}}$ & & & & & $-\bar{m}$ & $m \widehat{m}$ & & \\
\hline 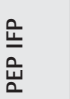 & 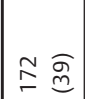 & & & & & & m & & & 的 $\overline{\hat{\sigma}}$ & & & & 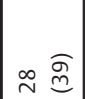 & & $\approx \widehat{N}$ & & $\wedge \stackrel{\widetilde{n}}{n}$ \\
\hline 莣 & 孛 $\widehat{\sigma}$ & & & & & & 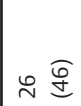 & & & nू 吕 & & $\bar{\sigma} \stackrel{\widehat{\infty}}{\infty}$ & & 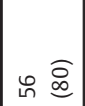 & & $\forall \widehat{\mathscr{g}}$ & & $=\stackrel{\sqrt[\Omega]{n}}{n}$ \\
\hline 产 & $\tilde{\sigma} \underset{\tilde{\sigma}}{\widetilde{E}}$ & & & & $\bar{\sim} \widetilde{\widehat{N}}$ & & จ & & & 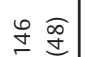 & & $\bar{\sigma} \stackrel{\infty}{\stackrel{\infty}{\infty}}$ & & 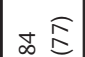 & 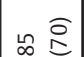 & $8 \underset{\infty}{\stackrel{\widehat{\infty}}{\infty}}$ & & $\infty \stackrel{\sqrt[\Omega]{n}}{\simeq}$ \\
\hline$\frac{\frac{i}{2}}{2}$ & $\tilde{\tilde{\gamma}} \overline{\underline{q}}$ & & 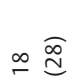 & in $\stackrel{\mathcal{F}}{=}$ & 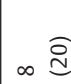 & $\stackrel{\stackrel{\circ}{\rho}}{=}$ & F寻 & $\sigma \widehat{\tilde{m}}$ & & $\stackrel{\leftrightarrow}{n} \stackrel{\widehat{\infty}}{=}$ & $\approx \stackrel{\stackrel{\sim}{\Xi}}{\stackrel{D}{a}}$ & & & $\therefore \stackrel{\widehat{\Omega}}{0}$ & & 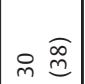 & 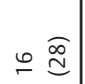 & 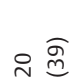 \\
\hline $\begin{array}{l}\sum_{0} \\
\vdots \\
\dot{z}\end{array}$ & $\mid \begin{array}{ll}\infty & \widehat{\sigma} \\
\sigma & 0 \\
\end{array}$ & & $g \widehat{E}$ & 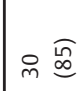 & $\pm \stackrel{\widehat{\Omega}}{\tilde{c}}$ & $\infty \stackrel{\widetilde{\Omega}}{\widetilde{c}}$ & 号 & $\stackrel{0}{0}$ & & $g \stackrel{\widehat{m}}{\tilde{c}}$ & $\tilde{0} \widehat{\widetilde{E}}$ & $g \stackrel{\widehat{\partial}}{=}$ & & $尺$ 妥 & & $f \overline{\hat{\theta}}$ & $q \bar{E}$ & $\bar{m}$ \\
\hline 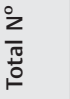 & $\begin{array}{l}\circ \\
\stackrel{0}{\circ}\end{array}$ & 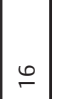 & ఫే & $\stackrel{n}{m}$ & q & $\stackrel{\text { ஜn }}{\stackrel{2}{n}}$ & ○ & $\hat{\imath}$ & ก & $\stackrel{\infty}{\sim}$ & $\infty$ & 8 & & $\stackrel{8}{\circ}$ & $\stackrel{\overbrace{}}{\simeq}$ & ミ & in & in \\
\hline 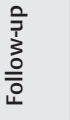 & 望 & 点 & 号 & 总 & 总 & 总 & $\stackrel{\mathscr{\vartheta}}{\mathscr{\checkmark}}$ & : & 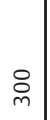 & 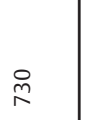 & 总 & 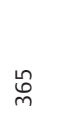 & த. & 总 & 吕 & 总 & 怘 & 䀫 \\
\hline هั & $\stackrel{m}{\grave{n}}$ & 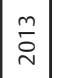 & $\stackrel{m}{\stackrel{n}{N}}$ & $\stackrel{m}{\grave{D}}$ & $\stackrel{+}{\stackrel{D}{N}}$ & $\stackrel{+}{\grave{n}}$ & $\stackrel{+}{\grave{n}}$ & $\stackrel{\vec{N}}{\stackrel{N}{N}}$ & $\stackrel{+}{\grave{n}}$ & $\stackrel{n}{\stackrel{n}{N}}$ & 圆 & $\stackrel{0}{\stackrel{N}{N}}$ & $\stackrel{0}{\stackrel{2}{N}}$ & $\begin{array}{l}0 \\
\stackrel{2}{\tilde{N}}\end{array}$ & 号 & $\begin{array}{l}0 \\
\stackrel{2}{N}\end{array}$ & 号 & $\stackrel{\hat{D}}{N}$ \\
\hline 总 & 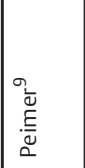 & 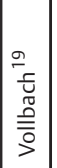 & 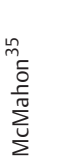 & 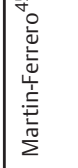 & 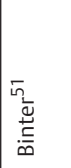 & 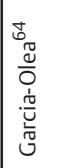 & 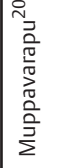 & $\begin{array}{l}0 \\
\stackrel{m}{\overline{0}} \\
\vdots \\
\vdots\end{array}$ & 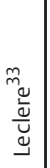 & 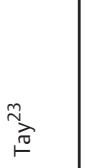 & 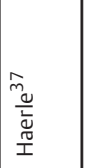 & 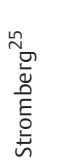 & 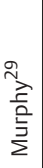 & 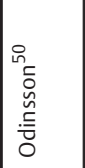 & 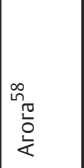 & 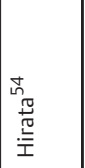 & 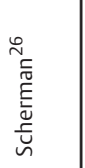 & 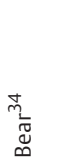 \\
\hline
\end{tabular}




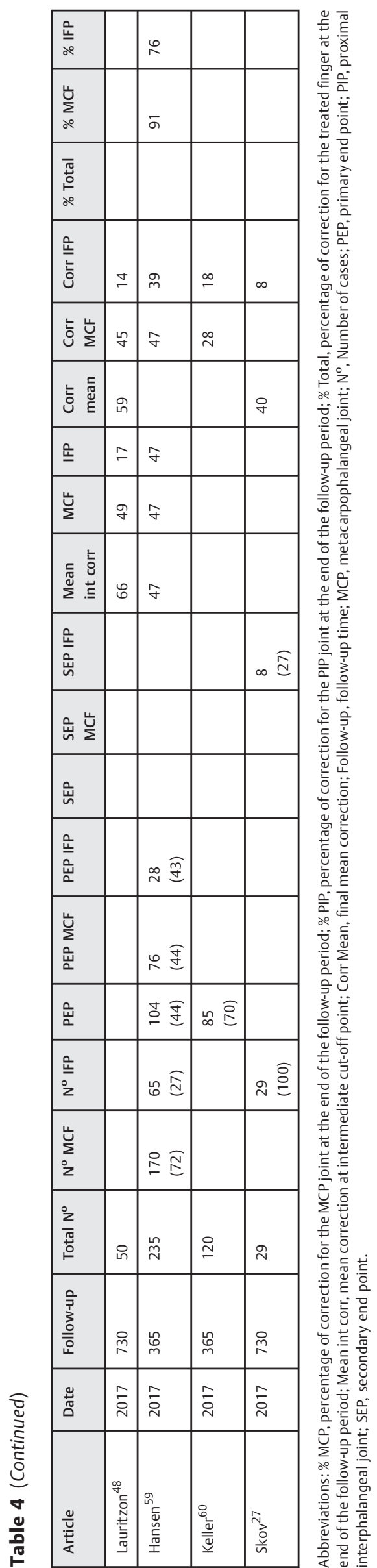

recurrences occurred after the first 3 years of treatment ${ }^{10}$ and are significantly minor regarding subsequent progression.

\section{Treatment Failures}

A concept not adequately clarified in the literature regarding $\mathrm{CCH}$ treatment is treatment failures, that is, the injection of $\mathrm{CCH}$ into a Dupuytren cord with no effect. Peimer, ${ }^{10}$ at the 5year follow-up of the CORDLESS study, indicates that the rate of patients treated ineffectively in the CORD and JOINT studies was $18 \%$ (9\% of MCPs and $32 \%$ of PIPs), whereas among the patients who completed the follow-up for the CORDLESS study, the rate was $14 \%$ (7\% of MCPs and $26 \%$ of PIPs). Only two more authors have covered this concept: Keller ${ }^{60}$ indicates a $3 \%$ rate of treatment failures, and Peimer (ref), in another study that does not include patients from the CORD studies, reports a rate of $16 \%$ (8 patients).

\section{Discussion}

Collagenase clostridium histolyticum is currently a therapeutic alternative for DD both in Europe ${ }^{8}$ and the USA. ${ }^{7}$ However, the heterogeneity of publications has been more the norm rather than the exception in studies related to $\mathrm{CCH}$. Even though virtually all studies have maintained a standard minimum contracture with which to begin the treatment (20 degrees), some have slightly increased this to 30 degrees. Other data, such as the loss of extension limit (initially set at 100 degrees for the finger), the variation in severity criteria with regard to adoption of own criteria rather than any other DD classification, show the heterogeneity in publications, as is common in publications related to Dupuytren surgery. ${ }^{13}$

The measurement of results is another example: the $\mathrm{CCH}$ treatment is performed at a joint level in an isolated manner; with the rupture of the cord, a compensatory correction of the adjacent joint of the same finger can be produced. Measuring both joints together after the treatment of only one of them constitutes an error that causes bias in clinical studies, as the correction of a joint can be masked by the affectation or the retraction of the adjacent finger. ${ }^{62}$ Likewise, the inclusion of untreated joints in the results, when they present baseline contracture, worsens the final result if a joint finger assessment (MCP + PIP) is done. In fact, to mitigate these shortcomings, in CORD studies the results are presented in two modes: on the one hand in terms of the correction of the treated joint, and on the other in the form of "range of movement" (ROM). ${ }^{6,14,54}$ Different forms of measurement (ROM, passive extension deficit [PED], isolated joints) are adopted in the studies, which complicate the comparability of results between the studies ( $\mathbf{- T a b l e ~} \mathbf{1}$ ).

Clinical results indicate that full extension of the fingers has been achieved in $~ 50 \%$ of the cases maintained for a year, and the mean reduction in contracture for all patients is at around $75 \%$, indicating a remarkable effectiveness of the treatment. The results of our study may seem contradictory with a higher reduction in digital contracture in studies lasting over 1 year than in studies covering less than 
1 year of progress, but the heterogeneity of published papers and the lack of homogeneity between the protocols used $^{63}$ explain this variation, which does not invalidate the final result. The fact that a greater number of patients have reached the primary end point in studies lasting more than 1 year is due, in part, to the fact that many of these studies have been funded by or related to the company that markets the drug, which is why the patient selection criteria, the surgeon's experience or the protocol for patient maintenance, minimizing the losses of individuals with a positive result, may be regarded as biased. $9,34,35,37,48,54,60$

With regard to joint treatment, the demonstrated results concur with many of the series published. The outcome for the MCP joint is better than the outcome for the PIP, and the recurrences of the latter are also much more frequent. The data presented does not allow for a study of the severity of the affected joint as some authors do ${ }^{9,14,46,57}$; the analyzed data indicate that a more severe initial contracture signifies a worse outcome for the treatment, more specifically for the PIP. With respect to the issue of recurrence, the lack of studies in the medium and long term prevents an objective assessment; however, the CORDLESS studies show a clear tendency toward recurrence with an overall rate of $48 \%$ at 5 years. ${ }^{10}$ Garcia-Olea ${ }^{64}$ quantifies the deterioration of the patients in their series at 1.5 degrees per month.

Physiotherapy protocols have not been included in clinical trials; thus, the improvement or sustainability of treatment with them cannot be assessed. Although the use of night splints is indicated, many studies have avoided this adjunctive measure, possibly due to poor compliance of the patients, ${ }^{41}$ and the discomfort it causes over an extended period of time. These two measures, without a doubt, are the most variable in the studies analyzed. One of the first comparative clinical studies ${ }^{32}$ actually assessed the use of these measures for treatment with $\mathrm{CCH}$ and concluded that their apparent benefit is in the short term.

Among the main problems that are not taken into account in the analyzed series is the rate of poor and "non-effective" outcomes. The same analysis is not performed on these patients as it is performed on those with good outcomes. There is currently no explanation as to why some patients do not respond to the treatment.

Among the limitations of our study, the main one is the possibility of biases in terms of data collection. Injection on a joint and the measurement of the full result on the affected finger is in itself a bias that some of the cited papers comment on, and it has made it difficult to compare this technique with the surgical procedures (partial fasciectomy), where traditionally the whole radius is treated. The results are usually expressed as a difference in degrees between the initial and final results with a range in each value. While obtaining the difference between both results for the assessment of the correction achieved is very simple, it is practically impossible to evaluate the standard deviation of the sample. This prevents large-scale statistical studies from being conducted, and thus constitutes the main limitation of our study. Unfortunately, these limitations are insurmountable and evident in the analysis of bibliography. $^{13}$
In conclusion, we can say that there is a lack of uniformity in the approach of the studies and in their outcomes with regard to the assessment of DD treatment with $\mathrm{CCH}$. Despite this issue, the results indicate a satisfactory response to treatment in a large number of patients maintained in the short and medium term, with a recurrence rate of over $50 \%$, occurring mainly within the first 3 years of follow-up. The exact recurrence rate is uncertain given the available data.

\section{Conflicts of Interest}

Authors declare that there were no conflict of interests for the writing of the present work.

\section{References}

1 Smeraglia F, Del Buono A, Maffulli N. Collagenase clostridium histolyticum in Dupuytren's contracture: a systematic review. $\mathrm{Br}$ Med Bull 2016;118(01):149-158

2 Desai SS, Hentz VR. The treatment of Dupuytren disease. J Hand Surg Am 2011;36(05):936-942

3 Hueston JT. Enzymic fasciotomy. Hand 1971;3(01):38-40

4 Badalamente MA, Hurst LC, Hentz VR. Collagen as a clinical target: nonoperative treatment of Dupuytren's disease. J Hand Surg Am 2002;27(05):788-798

5 Badalamente MA, Hurst LC. Enzyme injection as nonsurgical treatment of Dupuytren's disease. J Hand Surg Am 2000;25 (04):629-636

6 Hurst LC, Badalamente MA, Hentz VR, et al; CORD I Study Group. Injectable collagenase clostridium histolyticum for Dupuytren's contracture. N Engl J Med 2009;361(10):968-979

7 Zhao JZ, Hadley S, Floyd E, Earp BE, Blazar PE. The Impact of Collagenase Clostridium histolyticum Introduction on Dupuytren Treatment Patterns in the United States. J Hand Surg Am 2016;41 (10):963-968

8 Marks M, Krefter C, Herren DB. Treatment Methods for Patients with Dupuytren's Disease in Switzerland. Handchir Mikrochir Plast Chir 2016;48(03):155-160

9 Peimer CA, Blazar P, Coleman S, et al. Dupuytren contracture recurrence following treatment with collagenase clostridium histolyticum (CORDLESS study): 3-year data. J Hand Surg Am 2013;38 (01):12-22

10 Peimer CA, Blazar P, Coleman S, Kaplan FTD, Smith T, Lindau T. Dupuytren Contracture Recurrence Following Treatment With Collagenase Clostridium histolyticum (CORDLESS [Collagenase Option for Reduction of Dupuytren Long-Term Evaluation of Safety Study]): 5-Year Data. J Hand Surg Am 2015;40(08): 1597-1605

11 Badalamente MA, Hurst LC, Benhaim P, Cohen BM. Efficacy and safety of collagenase clostridium histolyticum in the treatment of proximal interphalangeal joints in dupuytren contracture: combined analysis of 4 phase 3 clinical trials. J Hand Surg Am 2015;40 (05):975-983

12 Witthaut J, Bushmakin AG, Gerber RA, Cappelleri JC, Le GraverandGastineau M-PH. Determining clinically important changes in range of motion in patients with Dupuytren's Contracture: secondary analysis of the randomized, double-blind, placebo-controlled CORD I study. Clin Drug Investig 2011;31(11):791-798

13 Warwick D. Dupuytren's disease: my personal view. J Hand Surg Eur Vol 2017;42(07):665-672

14 Gilpin D, Coleman S, Hall S, Houston A, Karrasch J, Jones N. Injectable collagenase Clostridium histolyticum: a new nonsurgical treatment for Dupuytren's disease. J Hand Surg Am 2010;35 (12):2027-38.e1 
15 Witthaut J, Jones G, Skrepnik N, Kushner H, Houston A, Lindau TR. Efficacy and safety of collagenase clostridium histolyticum injection for Dupuytren contracture: short-term results from 2 openlabel studies. J Hand Surg Am 2013;38(01):2-11

16 Warwick D, Arner M, Pajardi G, et al; POINT X Investigators. Collagenase clostridium histolyticum in patients with Dupuytren's contracture: results from POINT X, an open-label study of clinical and patient-reported outcomes. J Hand Surg Eur Vol 2015; 40(02):124-132

17 Gaston RG, Larsen SE, Pess GM, et al. The Efficacy and Safety of Concurrent Collagenase Clostridium Histolyticum Injections for 2 Dupuytren Contractures in the Same Hand: A Prospective, Multicenter Study. J Hand Surg Am 2015;40(10):1963-1971

18 Spanholtz TA, Holzbach T, Wallmichrath J, et al. [Treatment of Dupuytren's contracture by means of injectable collagenase: first clinical experiences]. Handchir Mikrochir Plast Chir Organ Deutschsprachigen Arbeitsgemeinschaft Handchir Organ Deutschsprachigen Arbeitsgemeinschaft Mikrochir Peripher Nerven Gefasse Organ V. 2011;43(05):275-280

19 Vollbach FH, Walle L, Fansa H. [Dupuytren's disease - patient satisfaction and functional results one year after partial fasciectomy and injection of collagenase]. Handchir Mikrochir Plast Chir 2013;45(05):258-264

20 Muppavarapu RC, Waters MJ, Leibman MI, Belsky MR, Ruchelsman DE. Clinical outcomes following collagenase injections compared to fasciectomy in the treatment of Dupuytren's contracture. Hand (NY) 2015; 10(02):260-265

21 Povlsen B, Povlsen SD. What is the better treatment for single digit dupuytren's contracture: surgical release or collagenase clostridium histolyticum (Xiapex) injection? Hand Surg 2014;19(03): 389-392

22 Zhou C, Hovius SER, Slijper HP, et al. Collagenase Clostridium Histolyticum versus Limited Fasciectomy for Dupuytren's Contracture: Outcomes from a Multicenter Propensity Score Matched Study. Plast Reconstr Surg 2015;136(01):87-97

23 Tay TKW, Tien H, Lim EYL. Comparison between Collagenase Injection and Partial Fasciectomy in the Treatment of Dupuytren's Contracture. Hand Surg 2015;20(03):386-390

24 Nydick JA, Olliff BW, Garcia MJ, Hess AV, Stone JD. A comparison of percutaneous needle fasciotomy and collagenase injection for dupuytren disease. J Hand Surg Am 2013;38(12):2377-2380

25 Strömberg J, Ibsen-Sörensen A, Fridén J. Comparison of Treatment Outcome After Collagenase and Needle Fasciotomy for Dupuytren Contracture: A Randomized, Single-Blinded, Clinical Trial With a 1-Year Follow-Up. J Hand Surg Am 2016;41(09): 873-880

26 Scherman P, Jenmalm P, Dahlin LB. One-year results of needle fasciotomy and collagenase injection in treatment of Dupuytren's contracture: A two-centre prospective randomized clinical trial. J Hand Surg Eur Vol 2016;41(06):577-582

27 Skov ST, Bisgaard T, Søndergaard P, Lange J. Injectable Collagenase Versus Percutaneous Needle Fasciotomy for Dupuytren Contracture in Proximal Interphalangeal Joints: A Randomized Controlled Trial. J Hand Surg Am 2017;42(05):321-328.e3

28 Sanjuan Cerveró R, Franco Ferrando N, Poquet Jornet J. Use of resources and costs associated with the treatment of Dupuytren's contracture at an orthopedics and traumatology surgery department in Denia (Spain): collagenase clostridium hystolyticum versus subtotal fasciectomy. BMC Musculoskelet Disord 2013; 14(01):293

29 Murphy LE, Murphy KM, Kilpatrick SM, Thompson NW. The use of Collagenase Clostridium Histolyticum in the management of Dupuytren's contracture-outcomes of a pilot study in a District General Hospital setting. Ulster Med J 2017;86(02):94-98

30 Sood A, Therattil PJ, Paik AM, Simpson MF, Lee ES. Treatment of dupuytren disease with injectable collagenase in a veteran population: a case series at the department of veterans affairs new jersey health care system. Eplasty 2014;14:e13
31 Waters MJ, Belsky MR, Blazar PE, Leibman MI, Ruchelsman DE. Collagenase enzymatic fasciotomy for Dupuytren contracture in patients on chronic immunosuppression. Am J Orthop 2015;44 (11):518-521

32 Skirven TM, Bachoura A, Jacoby SM, Culp RW, Osterman AL. The effect of a therapy protocol for increasing correction of severely contracted proximal interphalangeal joints caused by dupuytren disease and treated with collagenase injection. J Hand Surg Am 2013;38(04):684-689

33 Leclère F-M, Mathys L, Vögelin E. [Collagenase injection in Dupuytren's disease, evaluation of the ultrasound assisted technique]. Chir Main 2014;33(03):196-203

34 Bear BJ, Peimer CA, Kaplan FTD, Kaufman GJ, Tursi JP, Smith T. Treatment of Recurrent Dupuytren Contracture in Joints Previously Effectively Treated With Collagenase Clostridium histolyticum. J Hand Surg Am 2017;42(05):391.e1-391.e8

35 McMahon HA, Bachoura A, Jacoby SM, Zelouf DS, Culp RW, Osterman AL. Examining the efficacy and maintenance of contracture correction after collagenase clostridium histolyticum treatment for Dupuytren's disease. Hand (NY) 2013;8(03):261-266

36 Peimer CA, Skodny P, Mackowiak JI. Collagenase clostridium histolyticum for dupuytren contracture: patterns of use and effectiveness in clinical practice. J Hand Surg Am 2013;38(12): 2370-2376

37 Haerle M, Witthaut J, Giunta RE, et al. Treatment of Dupuytreńs contracture with collagenase clostridium histolyticum under clinical practice conditions: ReDUCTo study. Ger Plast Reconstr Aesthet Surg 2015;5:Doc06

38 Warwick DJ, Graham D, Worsley P. New insights into the immediate outcome of collagenase injections for Dupuytren's contracture. J Hand Surg Eur Vol 2016;41(06):583-588

39 Verheyden JR. Early outcomes of a sequential series of 144 patients with Dupuytren's contracture treated by collagenase injection using an increased dose, multi-cord technique. J Hand Surg Eur Vol 2015;40(02):133-140

40 Mickelson DT, Noland SS, Watt AJ, Kollitz KM, Vedder NB, Huang JI. Prospective randomized controlled trial comparing 1- versus 7day manipulation following collagenase injection for dupuytren contracture. J Hand Surg Am 2014;39(10):1933-1941.e1

41 Manning CJ, Delaney R, Hayton MJ. Efficacy and tolerability of Day 2 manipulation and local anaesthesia after collagenase injection in patients with Dupuytren's contracture. J Hand Surg Eur Vol 2014;39(05):466-471

42 Kaplan FTD, Badalamente MA, Hurst LC, Merrell GA, Pahk R. Delayed manipulation after collagenase clostridium histolyticum injection for Dupuytren contracture. Hand (NY) 2015;10(03): 578-582

43 Mármol-Soler S, Espejo-Ortega L, Gutiérrez-Ortega C, et al. Tratamiento no quirúrgico de la contractura de Dupuytren con colagenasa de Clostridium hystoliticum. Cir Plástica Ibero-Latinoam. 2013;39(03):247-254

44 Alberton F, Corain M, Garofano A, et al. Efficacy and safety of collagenase Clostridium histolyticum injection for Dupuytren contracture: report of 40 cases. Musculoskelet Surg 2014;98 (03):225-232

45 Martín-Ferrero MÁ, Simón-Pérez C, Rodríguez-Mateos JI, GarcíaMedrano B, Hernández-Ramajo R, Brotat-García M. Treatment of Dupuytren's disease using collagenase from Clostridium histolyticum. Rev Esp Cir Ortop Traumatol 2013;57(06):398-402

46 Coleman S, Gilpin D, Kaplan FTD, et al. Efficacy and safety of concurrent collagenase clostridium histolyticum injections for multiple Dupuytren contractures. J Hand Surg Am 2014;39(01): 57-64

47 Atroshi I, Nordenskjöld J, Lauritzson A, Ahlgren E, Waldau J, Waldén M. Collagenase treatment of Dupuytren's contracture using a modified injection method: a prospective cohort study of skin tears in 164 hands, including short-term outcome. Acta Orthop 2015;86(03):310-315 
48 Lauritzson A, Atroshi I. Collagenase injections for Dupuytren's disease: prospective cohort study assessing 2-year treatment effect durability. BMJ Open 2017;7(03):e012943

49 Grandizio LC, Akoon A, Heimbach J, Graham J, Klena JC. The Use of Residual Collagenase for Single Digits With Multiple-Joint Dupuytren Contractures. J Hand Surg Am 2017;42(06):472.e1-472. e6

50 Odinsson A, Brenne LE, Lurie TB, Finsen V. Dupuytren's Contracture. The Safety and Efficacy of Collagenase Treatment. J Hand Surg Asian Pac Vol 2016;21(02):187-192

51 Binter A, Neuwirth M, Rab M. [Treatment of Dupuytren's disease with collagenase - a 1-year follow-up of 37 patients]. Handchir Mikrochir Plast Chir 2014;46(06):355-360

52 Considine S, Heaney R, Hirpara KM. Early results of the use of collagenase in the treatment of Dupuytren's contracture. Ir J Med Sci 2015;184(02):323-327

53 Pérez-Giner RA, Aguilella L. Resultado precoz del tratamiento de la enfermedad de Dupuytren mediante inyección de colagenasa. Rev Iberoam Cir Mano. 2015;43(01):13-19

54 Hirata H, Tanaka K, Sakai A, Kakinoki R, Ikegami H, Tateishi N. Efficacy and safety of collagenase Clostridium histolyticum injection for Dupuytren's contracture in non-Caucasian Japanese patients (CORD-J Study): the first clinical trial in a non-Caucasian population. J Hand Surg Eur Vol 2016:1753193416653249

55 Verstreken F, Degreef I, Decramer A, et al. Effectiveness and safety of collagenase Clostridium histolyticum in Dupuytren's disease: an observational study in Belgium. Acta Orthop Belg 2016;82(02):397-404

56 European Medicines Agency - Find medicine - Xiapex.

57 Coleman S, Gilpin D, Tursi J, Kaufman G, Jones N, Cohen B. Multiple concurrent collagenase clostridium histolyticum injections to
Dupuytren's cords: an exploratory study. BMC Musculoskelet Disord 2012;13(01):61

58 Arora R, Kaiser P, Kastenberger T-J, Schmiedle G, Erhart S, Gabl M. Injectable collagenase Clostridium histolyticum as a nonsurgical treatment for Dupuytren's disease. Oper Orthop Traumatol 2016; 28(01):30-37

59 Hansen KL, Werlinrud JC, Larsen S, Ipsen T, Lauritsen J. Difference in Success Treating Proximal Interphalangeal and Metacarpophalangeal Joints with Collagenase: Results of 208 Treatments. Plast Reconstr Surg Glob Open 2017;5(04):e1275

60 Keller M, Arora R, Schmiedle G, Kastenberger T. Therapie des Morbus Dupuytren mit Kollagenase Clostridium histolyticum. Orthop 2017;1(07):321-327

61 Malafa MM, Lehrman C, Criley JW, Amirlak B. Collagenase Dupuytren Contracture: Achieving Single Treatment Success with a Hand Therapist-Based Protocol. Plast Reconstr Surg Glob Open 2016;4(02):e629

62 Rodrigues JN, Zhang W, Scammell BE, Davis TR. Dynamism in Dupuytren's contractures. J Hand Surg Eur Vol 2015;40(02):166-170

63 Sanjuan-Cerveró R, Carrera-Hueso FJ, Vazquez-Ferreiro P, GomezHerrero D. Adverse Effects of Collagenase in the Treatment of Dupuytren Disease: A Systematic Review. BioDrugs 2017;31(02): 105-115

64 García-Olea A, Dudley AF, Gimeno Andrade MD, Ocampos M, Fahandezh-Saddi H, Del Cerro M. Efectividad del tratamiento de la enfermedad de Dupuytren (ED) mediante fasciotomía enzimática con colagenasa. Estudio multicéntrico cohortes prospectivo. Rev Iberoam Cir Mano. 2014;42:97-106

65 Peimer CA, McGoldrick CA, Kaufman G. Nonsurgical Treatment of Dupuytren Contracture: 3-Year Safety Results Using Collagenase Clostridium histolyticum. J Hand Surg Am 2013;38(10):e52 


\begin{tabular}{|c|c|c|c|c|c|c|c|c|c|c|c|c|c|c|c|c|c|c|c|}
\hline$\frac{\rho}{\frac{2}{2}}$ & ఫु & $\begin{array}{l}0 \\
\text { in }\end{array}$ & & $\stackrel{m}{\stackrel{m}{r}}$ & $\varnothing$ & & $\infty$ & নু & & & & & in & 每 & $\stackrel{\infty}{+}$ & & & 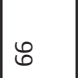 & \\
\hline 이 & $\infty$ & 旾 & & $\bar{\infty}$ & $\bar{\infty}$ & & & 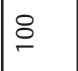 & & & & & $\infty$ & $\underbrace{}_{\infty}$ & 号 & & & $\infty$ & \\
\hline 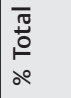 & 9 & 年 & & $\hat{\Omega}$ & $\stackrel{\operatorname{Ln}}{\wedge}$ & & & $\infty$ & & & & & i & 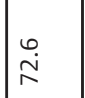 & $\mid \begin{array}{l}n \\
\stackrel{g}{g} \\
g\end{array}$ & & & & \\
\hline 氞亚 & I & $\approx$ & $\approx$ & $\bar{m}$ & $\stackrel{J}{m}$ & & g & & $\stackrel{\check{N}}{\sim}$ & $1 \hat{0}$ & 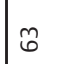 & $\stackrel{ \pm}{\sim}$ & m & $\stackrel{\Perp}{N}$ & $\stackrel{\infty}{+}$ & i & in & $\grave{\sim}$ & $\bar{N}$ \\
\hline 䓌 & F & $f$ & $m$ & 穴 & 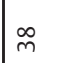 & & & f & f & $F$ & 号 & i & F & 占 & 多 & ڤ̇ & $\stackrel{\infty}{m}$ & $\approx$ & $\stackrel{\infty}{\sim}$ \\
\hline ¿ & $\begin{array}{l}\hat{b} \\
\dot{m}\end{array}$ & 离 & $m$ & $\hat{\text { iे }}$ & & $m$ & & Ұ & $\bar{\gamma}$ & 8 & & & & & & & & in & \\
\hline $\begin{array}{l}\frac{\hat{\underline{u}}}{\underline{u}} \\
\underline{u}\end{array}$ & F $\bar{\sigma}$ & $\infty \widehat{\mathbb{I}}$ & & & 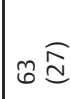 & & & & & & & & & $\pm \widehat{\bar{\Xi}}$ & $\simeq$ 尽 & & & & \\
\hline 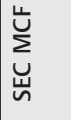 & $\bar{m} \stackrel{\widehat{\widetilde{d}}}{\stackrel{1}{d}}$ & $\wedge \stackrel{\sqrt[\Omega]{n}}{\Omega}$ & & & $\sigma \stackrel{\sqrt[\Omega]{n}}{=}$ & & & & & & & & & 홍 & 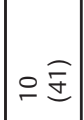 & & & & \\
\hline 岃 & 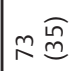 & 노송 & & & $\stackrel{\sim}{\stackrel{a}{\rightleftharpoons}}$ & & & & & & & & & $\stackrel{n}{\stackrel{\sigma}{\varrho}}$ & $\approx \stackrel{\widehat{o}}{+}$ & & & & \\
\hline $\begin{array}{l}\underline{\underline{u}} \\
\underline{\underline{u}} \\
\underline{\underline{u}}\end{array}$ & $\stackrel{\infty}{\sim} \stackrel{\bar{g}}{\mathbf{a}}$ & $\wedge \stackrel{\infty}{(}$ & & & 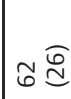 & & & ○ & & & & & & $\stackrel{\infty}{\stackrel{\infty}{\simeq}} \stackrel{0}{\simeq}$ & $\infty \stackrel{\widehat{\infty}}{\stackrel{0}{0}}$ & & & 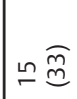 & \\
\hline 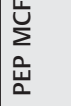 & $\stackrel{0}{\cong} \stackrel{0}{\circ}$ & $m$ 贫 & & & 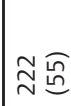 & & & 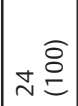 & & & & & & 总勇 & $\pm \stackrel{\widehat{D}}{\stackrel{0}{0}}$ & & & in $\stackrel{0}{=}$ & \\
\hline 㟔 & 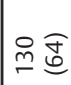 & 讨 & & & 离 & & & $\bar{m} \stackrel{\widehat{\circ}}{=}$ & & $\stackrel{\mathcal{N}}{\mathbb{I}}$ & & & & 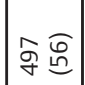 & $\approx \stackrel{\widehat{o}}{ \pm}$ & & & $\approx \widehat{O}$ & \\
\hline$\frac{\underline{a}}{2}$ & 영 & 兄 & & $\pm \stackrel{\widehat{\infty}}{\stackrel{c}{c}}$ & $\overline{\tilde{N}} \stackrel{\varrho}{\varrho}$ & & $\approx \stackrel{\bar{\Xi}}{=}$ & $\wedge \mathbb{\mathbb { Z }}$ & 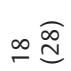 & 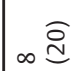 & n $\stackrel{\widehat{\sigma}}{=}$ & $\ln \stackrel{\widehat{\sigma}}{=}$ & 的宮 & 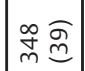 & $\approx \bar{q}$ & & $\circ \stackrel{0}{=}$ & 攻㣽 & \\
\hline$\sum_{z}^{\frac{U}{z}}$ & $\stackrel{m}{m} \underline{\underline{n}}$ & 过 & & $\approx \overline{\bar{\sigma}}$ & 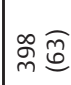 & & & $\underset{\sim}{\mathbb{E}}$ & o $\bar{g}$ & $\approx \widetilde{m}$ & 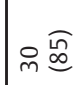 & $\pm \overline{\bar{y}}$ & - $\overline{\mathrm{m}}$ & $\overline{\tilde{r}} \widehat{\widehat{\sigma}}$ & $\underset{\sim}{\stackrel{\rho}{\Omega}}$ & & 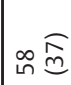 & 능 & \\
\hline $\begin{array}{l}\stackrel{\circ}{\circ} \\
\frac{\circ}{\pi} \\
\stackrel{0}{\circ}\end{array}$ & 穴 & 守 & $\div$ & $\stackrel{0}{n}$ & $\overparen{్}$ & $\bullet$ & $\approx$ & $\bar{m}$ & \& & q & $\stackrel{\sim}{m}$ & $\dot{m}$ & 6 & $\underset{\infty}{\stackrel{\Omega}{\infty}}$ & ๒ & $m$ & 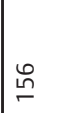 & 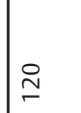 & ก \\
\hline 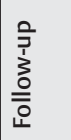 & 8 & 8 & $\Xi$ & 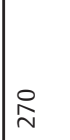 & m & $\begin{array}{l}n \\
\hat{0} \\
m\end{array}$ & i & i & 号 & $\stackrel{\infty}{\stackrel{\infty}{\circ}}$ & 点 & $\stackrel{\infty}{\circ}$ & $\stackrel{\infty}{\infty}$ & $\stackrel{尺}{N}$ & i & 年 & 点 & 8 & 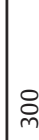 \\
\hline 苋 & 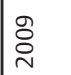 & 产 & i. & $\frac{\sim}{2}$ & $\stackrel{m}{i}$ & $\mid \begin{array}{l}m \\
\dot{n}\end{array}$ & 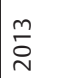 & $\stackrel{m}{\stackrel{m}{a}}$ & 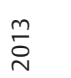 & 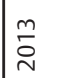 & 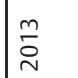 & $\stackrel{m}{\stackrel{n}{n}}$ & m & 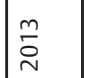 & 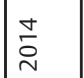 & 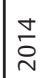 & 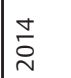 & $\frac{\text { N }}{\stackrel{2}{\circ}}$ & $\frac{\text { d }}{\dot{n}}$ \\
\hline 窇 & 蒙 & 高 & 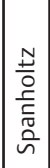 & 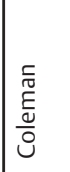 & 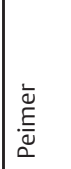 & $\mid \begin{array}{c}\tilde{y} \\
\tilde{D} \\
\overline{\overline{0}} \\
\mathcal{\rho}\end{array}$ & 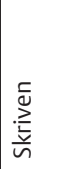 & 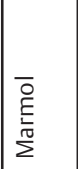 & 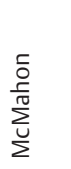 & 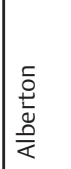 & 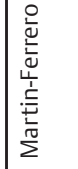 & 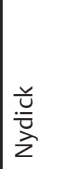 & 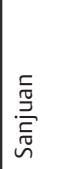 & 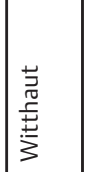 & 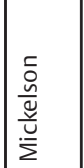 & 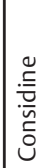 & 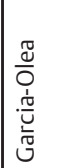 & 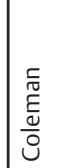 & 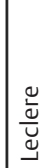 \\
\hline
\end{tabular}




\begin{tabular}{|c|c|c|c|c|c|c|c|c|c|c|c|c|c|c|c|c|c|c|c|}
\hline \begin{tabular}{|l} 
这 \\
응
\end{tabular} & & in & & 2 & & & & 8 & & $\tilde{m}$ & & & & & & $\infty$ & & & \\
\hline ஃ & & "ே & & $\infty$ & & & & $\Phi$ & & $\infty$ & & & & & & के & & & \\
\hline 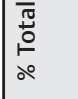 & & n & & & & & & i & & & & & & & & $\bar{\sigma}$ & $\bar{\infty}$ & & $\stackrel{n}{\wedge}$ \\
\hline 홍을 을 & 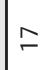 & $\frac{1}{4}$ & $m$ & $\bar{m}$ & m & $\stackrel{ \pm}{N}$ & & & $\stackrel{\infty}{\sim}$ & 9 & $q$ & i & & $\stackrel{\infty}{-}$ & $\ddot{\nu}$ & $\stackrel{m}{m}$ & & $\stackrel{\infty}{m}$ & $m$ \\
\hline نे & $\stackrel{2}{\sim}$ & g & F & m & in & $m$ & 夺 & & $\grave{m}$ & $m$ & $\stackrel{\infty}{m}$ & $\stackrel{\infty}{\sim}$ & in & 号 & $\tilde{m}$ & $\approx$ & & $\bar{t}$ & 兄 \\
\hline 空 & & & & & & & & $\stackrel{2}{R}$ & 只 & & $m$ & & in & & & q & 年 & q & 尔 \\
\hline 虽 & & & & & & $\pm \widetilde{\mathcal{I}}$ & & $\hat{\widetilde{N}} \widehat{\widetilde{J}}$ & & & & & & & & $m \stackrel{\varrho}{\varrho}$ & i $\widehat{\mathbb{E}}$ & & \\
\hline 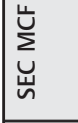 & & & & & & 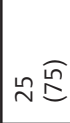 & & $\stackrel{\mathbb{N}}{\mathbb{d}}$ & & & & & & & & - 0 & $\approx \overline{\tilde{n}}$ & & \\
\hline 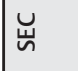 & & & & & & m & & 釆危 & & & & & & & & $m \widehat{\tilde{n}}$ & $\stackrel{\infty}{\sim} \stackrel{\sqrt[\Omega]{ }}{\Omega}$ & & \\
\hline $\begin{array}{l}\text { 音 } \\
\text { 㟧 }\end{array}$ & & & & 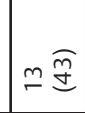 & in & & & 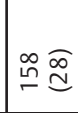 & & & & & & & $\stackrel{\infty}{\sim} \stackrel{\widehat{\rho}}{\stackrel{0}{0}}$ & $\approx \overline{\tilde{n}}$ & $\pm \stackrel{\mathscr{n}}{\mathscr{C}}$ & & \\
\hline 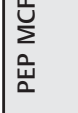 & & & & $\approx \widehat{N}$ & $\simeq \stackrel{\widehat{\circ}}{\varrho}$ & & $2 \frac{\pi}{2}$ & 周悉 & & & & & $\bar{\sigma} \stackrel{\widehat{\infty}}{\infty}$ & & 㶽高 & 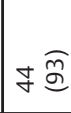 & 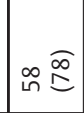 & & \\
\hline 产 & & & & & m $\stackrel{\widehat{\circ}}{\varrho}$ & & & 角 & & & & & $\bar{\sigma} \stackrel{\widehat{\infty}}{\infty}$ & & $\pm \underset{\infty}{\mathbb{E}}$ & $\begin{array}{l}\widehat{\nwarrow} \\
\infty \\
\varnothing \\
\varnothing\end{array}$ & 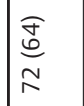 & $\begin{array}{l}0 \\
\stackrel{0}{0} \\
0 \\
0\end{array}$ & \\
\hline$\frac{\text { 을 }}{z}$ & & $\infty \stackrel{0}{=}$ & & $\underset{m}{\frac{\pi}{3}}$ & in $\stackrel{\widehat{\infty}}{\stackrel{0}{0}}$ & m & & 瓷总 & & 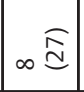 & $0 \stackrel{0}{+}$ & $\approx \stackrel{\varrho}{\varrho}$ & & & 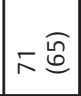 & 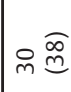 & $g \stackrel{0}{0}$ & $\wedge \stackrel{\widetilde{F}}{\Xi}$ & 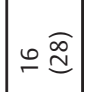 \\
\hline $\mid \sum_{z}^{u}$ & & ₹ $\stackrel{f}{\infty}$ & & $\infty_{m} \frac{\pi}{\frac{\pi}{3}}$ & $\simeq \widehat{\widetilde{\sigma}}$ & m & & 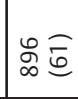 & & $\bar{\sim} \widehat{\mathbb{E}}$ & $\wedge \widehat{\hat{M}}$ & 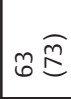 & $8 \stackrel{\widehat{\partial}}{=}$ & & 尺否 & $f \overline{\bar{\sigma}}$ & $\nabla \stackrel{\widehat{\theta}}{\circ}$ & 요 & क्ष \\
\hline 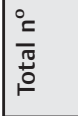 & 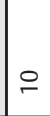 & in & $\bar{\sim}$ & & $m$ & & 8 & 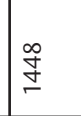 & 吕 & ก & $m$ & $\infty$ & 8 & & $\stackrel{9}{\circ}$ & & $\Xi$ & F & in \\
\hline 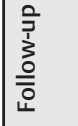 & & ø & $\stackrel{\infty}{\square}$ & 8 & 8 & 亲 & 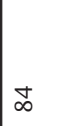 & ৪ & 8 & $\bar{\sim}$ & ì & 点 & 峉 & 呁 & 晜 & $\begin{array}{l}\text { L } \\
\text { n }\end{array}$ & ৪ & $\stackrel{\infty}{-}$ & 柋 \\
\hline 苛 & 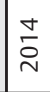 & 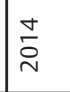 & 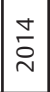 & $\underset{\sim}{\stackrel{T}{N}}$ & 吕 & $\stackrel{n}{\stackrel{n}{N}}$ & $\stackrel{n}{\stackrel{n}{n}}$ & $\stackrel{n}{\stackrel{n}{\sim}}$ & $\stackrel{\llcorner}{\stackrel{n}{\sim}}$ & $\stackrel{n}{\stackrel{n}{\sim}}$ & $\stackrel{n}{\stackrel{n}{n}}$ & $\stackrel{n}{\stackrel{n}{n}}$ & $\stackrel{0}{\stackrel{\sim}{\sim}}$ & $\begin{array}{l}0 \\
\stackrel{2}{2} \\
\end{array}$ & $\stackrel{0}{\stackrel{\sim}{\sim}}$ & 吕 & $\stackrel{\circ}{\stackrel{0}{2}}$ & $\stackrel{0}{\stackrel{0}{2}}$ & 产 \\
\hline 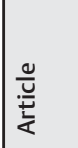 & 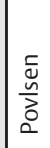 & 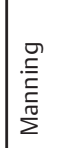 & 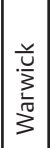 & 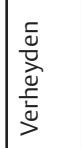 & 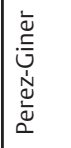 & 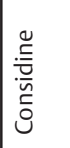 & $\frac{\partial}{\frac{D}{N}}$ & \begin{tabular}{|l}
$\frac{\sqrt{0}}{0}$ \\
$\frac{\sqrt{0}}{\underline{N}}$
\end{tabular} & 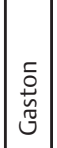 & 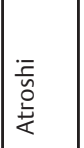 & 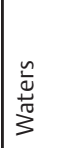 & 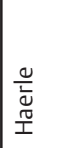 & 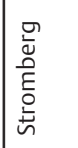 & $\mid \begin{array}{l}\text { 즐 } \\
\text { 言 } \\
2\end{array}$ & 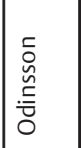 & 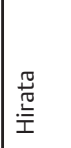 & 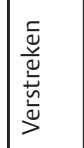 & $\frac{\frac{\pi}{0}}{\frac{\pi}{\pi}}$ & 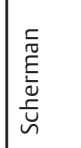 \\
\hline
\end{tabular}




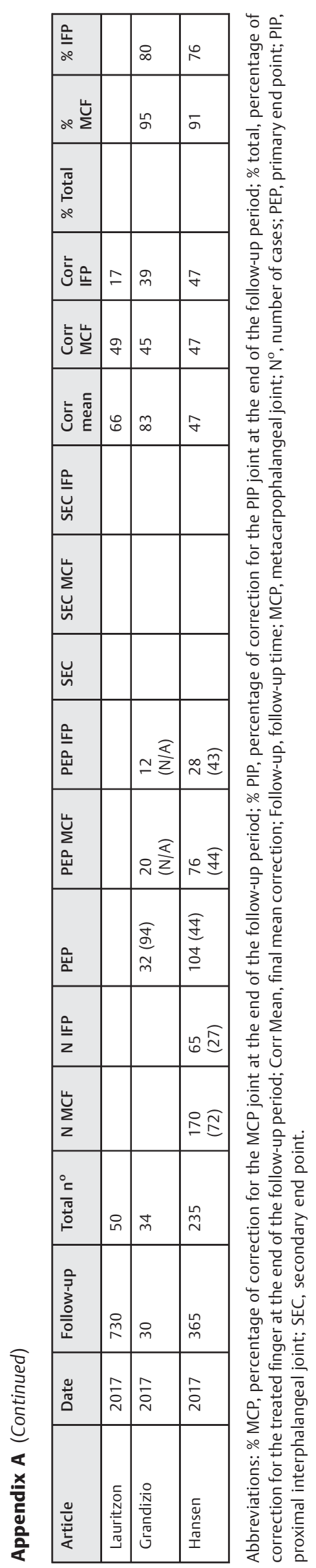

